\title{
THE SLOAN DIGITAL SKY SURVEY QUASAR CATALOG. III. THIRD DATA RELEASE
}

\author{
Donald P. Schneider, ${ }^{1}$ Patrick B. Hall, ${ }^{2,3}$ Gordon T. Richards, ${ }^{3}$ Daniel E. Vanden Berk, ${ }^{1}$ Scott F. Anderson, ${ }^{4}$
} Xiaohui Fan, ${ }^{5}$ Sebastian Jester, ${ }^{6}$ Chris Stoughton, ${ }^{6}$ Michael A. Strauss, ${ }^{3}$ Mark SubbaRao, ${ }^{7,8}$ W. N. Brandt, ${ }^{1}$ James E. Gunn, ${ }^{3}$ Brian Yanny, ${ }^{6}$ Neta A. Bahcall, ${ }^{3}$ J. C. Barentine, ${ }^{9}$ Michael R. Blanton, ${ }^{10}$ William N. Boroski, ${ }^{6}$ Howard J. Brewington, ${ }^{9}$ J. Brinkmann, ${ }^{9}$ Robert Brunner, ${ }^{11}$ István Csabai, ${ }^{12}$ Mamoru Doi, ${ }^{13}$ Daniel J. Eisenstein, ${ }^{5}$ Joshua A. Frieman, ${ }^{7}$ Masataka Fukugita, ${ }^{14,15}$ Jim Gray, ${ }^{16}$ Michael Harvanek, ${ }^{9}$ Timothy M. Heckman, ${ }^{17}$ Željko Ivezić, ${ }^{4}$

Stephen Kent, ${ }^{6}$ S. J. Kleinman, ${ }^{9}$ Gillian R. Knapp, ${ }^{3}$ Richard G. Kron, ${ }^{6,7}$ Jurek Krzesinski, ${ }^{9,} 18$ Daniel C. Long, ${ }^{9}$ Jon Loveday, ${ }^{19}$ Robert H. Lupton, ${ }^{3}$ Bruce Margon, ${ }^{20}$ Jeffrey A. Munn, ${ }^{21}$ Eric H. Neilsen, ${ }^{9}$ Heidi Jo Newberg, ${ }^{22}$ Peter R. Newman, ${ }^{9}$ R. C. Nichol, ${ }^{23}$ Atsuko Nitta, ${ }^{9}$ Jeffrey R. Pier, ${ }^{21}$ Constance M. Rockosi, ${ }^{24}$ David H. SaXe, ${ }^{15}$ David J. Schlegel, ${ }^{3,25}$ Stephanie A. Snedden, ${ }^{9}$ Alexander S. Szalay, ${ }^{17}$ Aniruddha R. Thakar, ${ }^{17}$ Alan Uomoto, ${ }^{26}$ Wolfgang Voges, ${ }^{27}$ and Donald G. York ${ }^{7,28}$

Received 2004 December 23; accepted 2005 March 28

\begin{abstract}
We present the third edition of the Sloan Digital Sky Survey (SDSS) Quasar Catalog. The catalog consists of the 46,420 objects in the SDSS Third Data Release that have luminosities larger than $M_{i}=-22$ (in a cosmology with $H_{0}=70 \mathrm{~km} \mathrm{~s}^{-1} \mathrm{Mpc}^{-1}, \Omega_{M}=0.3$, and $\Omega_{\Lambda}=0.7$ ), have at least one emission line with FWHM larger than $1000 \mathrm{~km} \mathrm{~s}^{-1}$ or are unambiguously broad absorption line quasars, are fainter than $i=15.0$, and have highly reliable redshifts. The area covered by the catalog is $\approx 4188 \mathrm{deg}^{2}$. The quasar redshifts range from 0.08 to 5.41 , with a median value of 1.47; the high-redshift sample includes 520 quasars at redshifts greater than 4, of which 17 are at redshifts greater than 5 . For each object the catalog presents positions accurate to better than $0.22 \mathrm{rms}$ per coordinate, five-band (ugriz) CCD-based photometry with typical accuracy of $0.03 \mathrm{mag}$, and information on the morphology and selection method. The catalog also contains radio, near-infrared, and X-ray emission properties of the quasars, when available, from other large-area surveys. The calibrated digital spectra cover the wavelength region 3800-9200 $\AA$ at a spectral resolution of $\simeq 2000$; the spectra can be retrieved from the public database using the information provided in the catalog. A total of 44,221 objects in the catalog were discovered by the SDSS; 28,400 of the SDSS discoveries are reported here for the first time.
\end{abstract}

Key words: catalogs — quasars: general — surveys

Online material: machine-readable table

\section{INTRODUCTION}

This paper describes the third edition of the Sloan Digital Sky Survey (SDSS; York et al. 2000) Quasar Catalog. The first two editions, coinciding with the SDSS Early Data Release (EDR; Stoughton et al. 2002) and the SDSS First Data Release (DR1; Abazajian et al. 2003), contained 3814 and 16,713 quasars, re-

\footnotetext{
${ }^{1}$ Department of Astronomy and Astrophysics, Pennsylvania State University, 525 Davey Laboratory, University Park, PA 16802; dps@astro.psu.edu.

2 Department of Physics and Astronomy, York University, 4700 Keele Street, Toronto, ON M3J 1P3, Canada.

${ }^{3}$ Princeton University Observatory, Princeton, NJ 08544

4 Department of Astronomy, University of Washington, Box 351580, Seattle, WA 98195.

5 Steward Observatory, University of Arizona, 933 North Cherry Avenue, Tucson, AZ 85721.

${ }^{6}$ Fermi National Accelerator Laboratory, P.O. Box 500, Batavia, IL 60510.

7 Astronomy and Astrophysics Center, University of Chicago, 5640 South Ellis Avenue, Chicago, IL 60637.

8 Adler Planetarium and Astronomy Museum, 1300 South Lake Shore Drive, Chicago, IL 60605.

9 Apache Point Observatory, P.O. Box 59, Sunspot, NM 88349-0059.

${ }^{10}$ Department of Physics, New York University, 4 Washington Place, New York, NY 10003.

${ }^{11}$ Department of Astronomy, University of Illinois, 1002 West Green Street, Urbana, IL 61801.

12 Department of Physics of Complex Systems, Eötvös Loránd University, Pf. 32, H-1518 Budapest, Hungary.

${ }^{13}$ Department of Astronomy and Research Center for the Early Universe, School of Science, University of Tokyo, Mitaka, Tokyo 181-0015, Japan.

${ }^{14}$ Institute for Cosmic Ray Research, University of Tokyo, Kashiwa 2778582, Japan.
}

spectively (Schneider et al. 2002, 2003, hereafter Paper I and Paper II, respectively). The current catalog contains 46,420 quasars from the SDSS Third Data Release (DR3; Abazajian et al. $2005)$, of which $28,400(61 \%)$ are presented here for the first time. The number of new quasars reported here is comparable to the number in the final 2dF QSO Redshift Survey (2QZ) catalog (Croom et al. 2004); the total size is similar to that of the NASA/ IPAC Extragalactic Database (NED) Quasar Catalog.

\footnotetext{
15 Institute for Advanced Study, Einstein Drive, Princeton, NJ 08540.

16 Microsoft Research, 455 Market Street, Suite 1690, San Francisco, CA 94105.

17 Department of Physics and Astronomy, The Johns Hopkins University, 3400 North Charles Street, Baltimore, MD 21218.

18 Mount Suhora Observatory, Cracow Pedagogical University, ul. Podchorazych 2, 30-084 Krakow, Poland.

19 Astronomy Centre, University of Sussex, Falmer, Brighton BN1 9QJ, UK.

20 Space Telescope Science Institute, 3700 San Martin Drive, Baltimore, MD 21218.

${ }^{21}$ US Naval Observatory, Flagstaff Station, P.O. Box 1149, Flagstaff, AZ 86002-1149.

22 Department of Physics, Applied Physics and Astronomy, Rensselaer Polytechnic Institute, Troy, NY 12180.

23 Institute of Cosmology and Gravitation, University of Portsmouth, Mercantile House, Hampshire Terrace, Portsmouth PO1 2EG, UK.

${ }^{24}$ UCO/Lick Observatory, University of California, Santa Cruz, CA 96064.

25 Lawrence Berkeley National Laboratory, Physics Division, 1 Cyclotron Road, Berkeley, CA 94720.

${ }^{26}$ Carnegie Observatories, 813 Santa Barbara Street, Pasadena, CA 91101.

27 Max-Planck-Institut für extraterrestrische Physik, Giessenbachstrasse 1, D-85741 Garching, Germany.

28 Enrico Fermi Institute, University of Chicago, 5640 South Ellis Avenue, Chicago, IL 60637.
} 
The catalog in the present paper consists of the DR3 objects that have luminosities larger than $M_{i}=-22.0$ (calculated assuming an $H_{0}=70 \mathrm{~km} \mathrm{~s}^{-1} \mathrm{Mpc}^{-1}, \Omega_{M}=0.3, \Omega_{\Lambda}=0.7$ cosmology [Spergel et al. 2003], which is used throughout this paper) and whose SDSS spectra contain at least one broad emission line (velocity FWHM larger than $\approx 1000 \mathrm{~km} \mathrm{~s}^{-1}$ ) or are unambiguously broad absorption line (BAL) quasars. The catalog also has a bright limit of $i=15.0$. The quasars range in redshift from 0.08 to 5.41 , and $44,221(95 \%)$ were discovered by the SDSS.

The objects are denoted in the catalog by their DR3 J2000.0 coordinates; the format for the object name is SDSS Jhhmmss.ss + ddmmss.s. Since continual improvements are being made to the SDSS data processing software, the astrometric solutions to a given set of observations can result in modifications to the coordinates of an object at the $0.1-0{ }^{\prime \prime} .2$ level; hence, the designation of a given source can change between data releases. Except on very rare occasions (see $\S 5.1$ ), this change in position is much less than $1^{\prime \prime}$. When merging SDSS quasar catalogs with previous databases one should always use the coordinates, not object names, to identify unique entries.

The DR3 catalog does not include classes of active galactic nuclei such as type II quasars, Seyfert galaxies, and BL Lacertae objects; studies of these sources in the SDSS can be found in Zakamska et al. (2003) for type II quasars, Kauffmann et al. (2003) and Hao et al. (2005) for Seyfert galaxies, and Anderson et al. (2003) and Collinge et al. (2005) for BL Lac objects. Spectra of the highest redshift SDSS quasars $(z>5.7$; e.g., Fan et al. 2003) were not acquired as part of the SDSS survey, so they are not included in the catalog.

The observations used to produce the catalog are presented in $\S 2$; the construction of the catalog and the catalog format are discussed in $\delta \S 3$ and 4, respectively. Section 5 contains an overview of the catalog, and a brief description of future work is given in $\S 6$. The catalog is presented in an electronic table in this paper and can also be found at an SDSS public Web site. ${ }^{29}$

\section{OBSERVATIONS}

\subsection{Sloan Digital Sky Survey}

The SDSS uses a CCD camera (Gunn et al. 1998) on a dedicated $2.5 \mathrm{~m}$ telescope at Apache Point Observatory, New Mexico, to obtain images in five broad optical bands (ugriz; Fukugita et al. 1996) over approximately 10,000 deg ${ }^{2}$ of the high Galactic latitude sky. The survey data processing software measures the properties of each detected object in the imaging data in all five bands and determines and applies both astrometric and photometric calibrations (Pier et al. 2003; Lupton et al. 2001; Ivezić et al. 2004). Photometric calibration is provided by simultaneous observations with a 20 inch $(0.5 \mathrm{~m})$ telescope at the same site (see Hogg et al. 2001; Smith et al. 2002; Stoughton et al. 2002). The SDSS photometric system is based on the AB magnitude scale (Oke \& Gunn 1983). The catalog contains photometry from 136 different SDSS imaging runs acquired between 1998 September 19 (run 94) and 2003 May 1 (run 3927) and spectra from 826 spectroscopic plates taken between 2000 March 5 and 2003 July 6.

\subsection{Target Selection}

The SDSS filter system was designed to identify quasars at redshifts between 0 and approximately 6 (see Richards et al. 2002); most quasar candidates are selected on the basis of their location in multidimensional SDSS color space. The point-spread

\footnotetext{
${ }^{29}$ See http://www.sdss.org/dr3/products/value_added/qsocat_dr3.html.
}

function (PSF) magnitudes are used for the quasar target selection, and the selection is based on magnitudes and colors that have been corrected for Galactic extinction (using the maps of Schlegel et al. 1998). An $i$ magnitude limit of 19.1 is imposed for candidates whose colors indicate a probable redshift of less than $\approx 3$ (selected from the ugri color cube); high-redshift candidates (selected from the griz color cube) are accepted if $i<20.2$. The errors on the $i$ measurements are typically $0.02-0.03$ and $0.03-$ 0.04 mag at the brighter and fainter limits, respectively. The SDSS images of the high-redshift candidates must be unresolved. In addition to the multicolor selection, unresolved objects brighter than $i=19.1$ that lie within 2".0 of a FIRST radio source (Becker et al. 1995) are also identified as primary quasar candidates. A detailed description of the quasar selection process and possible biases can be found in Richards et al. (2002) and Paper II.

Supplementing the primary quasar sample described above are quasars that were targeted by the following SDSS software selection algorithms: galaxy (Strauss et al. 2002; Eisenstein et al. 2001), X-ray (object near the position of a ROSAT All-Sky Survey [RASS; Voges et al. 1999, 2000] source; see Anderson et al. 2003), star (point source with unusual color), or serendipity (unusual color or FIRST matches). No attempt at completeness was made for the last three categories. Most of the DR3 quasars that fall below the magnitude limits of the quasar survey were selected by the serendipity algorithm (see $\S 5$ ). Target selection also imposes a maximum brightness limit $(i=15.0)$ on quasar candidates; the spectra of objects that exceed this brightness would contaminate the adjacent spectra on the detectors of the SDSS spectrographs.

One of the most important tasks during the SDSS commissioning period was to refine the quasar target selection algorithm (see Papers I and II); some of the DR3 data (and all the material in Paper II) were taken before the quasar selection algorithm as described in Richards et al. (2002) was implemented. Once the final target selection software was installed, the algorithm was applied to the entire SDSS photometric database, and the DR3 quasar catalog lists the selection target flag for each object produced by the final selection algorithm. Most of the quasars that have been added to the catalog since the DR1 version were found with the Richards et al. (2002) algorithm.

It is important to note that extreme care must be exercised when constructing statistical samples from this catalog; if one uses the values produced by only the latest version of the selection software, not only must one drop known quasars that were not identified as quasar candidates by the final selection software, one must also account for quasar candidates produced by the final version that were not observed in the SDSS spectroscopic survey (this can occur in regions of sky whose spectroscopic targets were identified by early versions of the selection software). The selection for the UV-excess quasars, which comprise the majority $(\approx 80 \%)$ of the objects in the DR3 catalog, has remained reasonably uniform; the changes to the selection algorithm were primarily designed to increase the effectiveness of the identification of $3.0<$ $z<3.8$ quasars. Extensive discussions of the completeness and efficiency of the selection can be found in Vanden Berk et al. (2005) and G. T. Richards et al. (2005, in preparation); the latter paper discusses the issues that are important for the construction of statistical SDSS quasar samples. The survey efficiency (the ratio of quasars to quasar candidates) for the ugri-selected candidates, which comprise the bulk of the quasar sample, is about $75 \%$.

\subsection{Spectroscopy}

Spectroscopic targets chosen by the various SDSS selection algorithms (i.e., quasars, galaxies, stars, and serendipity) are 

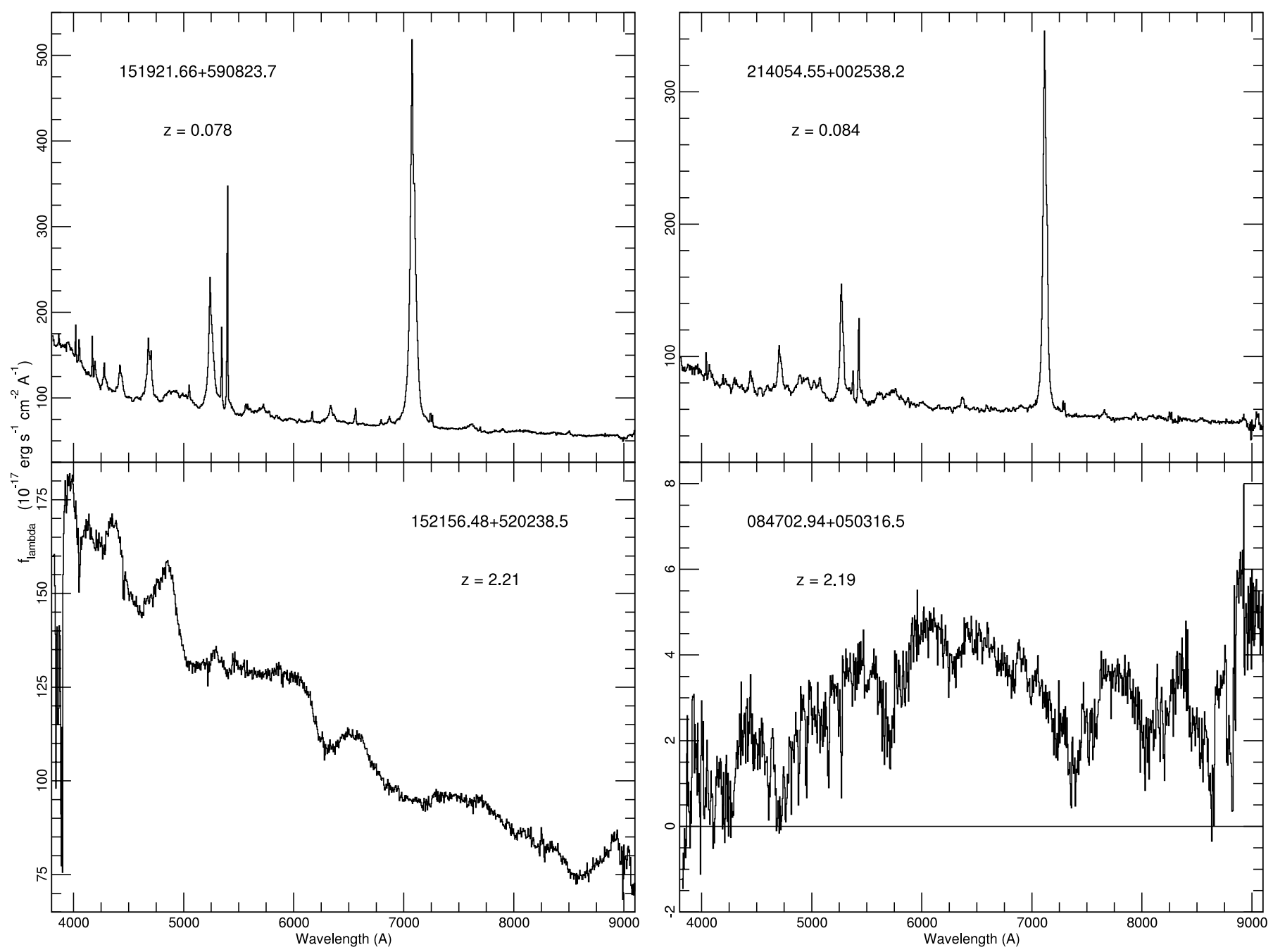

FIG. 1.-Example of data produced by the SDSS spectrographs. The spectral resolution of the data ranges from 1800 to 2100 ; a dichroic splits the beam at $6150 \AA$. The data have been rebinned to $5 \AA$ pixel $^{-1}$ for display purposes. All four of the quasars were discovered by the SDSS and are reported here for the first time. Notes on spectra: SDSS J151921.66+590823.7 and SDSS J214054.55+002538.2 are the lowest redshift quasars among the new objects; SDSS J152156.48+520238.5 is a moderate-redshift quasar that is both bright $(i=15.44)$ and extremely luminous $\left(M_{i}=-30.2\right)$; SDSS J084702.94+050316.5 is an example of a low-ionization BAL quasar with a red continuum and a strong $\mathrm{Mg}$ II absorption feature at $\approx 8900 \AA$.

arranged onto a series of $3^{\circ}$ diameter circular fields (Blanton et al. 2003). Details of the spectroscopic observations can be found in York et al. (2000), Castander et al. (2001), Stoughton et al. (2002), and Paper I. There are 826 DR3 spectroscopic fields; the locations of the plate centers can be found from the information given by Abazajian et al. (2005). The DR3 spectroscopic program attempted to cover, in a well-defined manner, an area of $\approx 4188 \mathrm{deg}^{2}$. Spectroscopic plate 716 was the first spectroscopic observation that was based on the final version of the quasar target selection algorithm (Richards et al. 2002); however, the detailed tiling information in the SDSS database must be consulted to identify those regions of sky targeted with the final selection algorithm.

The two double spectrographs produce data covering 3800$9200 \AA$ at a spectral resolution of $\simeq 2000$. The data, along with the associated calibration frames, are processed by the SDSS spectroscopic pipeline (see Stoughton et al. 2002). The calibrated spectra are classified into various groups (e.g., star, galaxy, quasar), and redshifts are determined by two independent software packages. Objects whose spectra cannot be classified by the software are flagged for visual inspection. Figure 1 shows the calibrated SDSS spectra of four previously unknown catalog quasars representing a range of properties.
The spectrophotometric calibration has been considerably improved since DR1; details of the changes are given in Abazajian et al. (2004). The processed DR3 spectra have not been corrected for Galactic extinction; this is a change from all previous SDSS data releases.

\section{CONSTRUCTION OF THE SDSS QUASAR CATALOG}

The quasar catalog was constructed in three stages: (1) creation of a quasar candidate database, (2) visual examination of the candidates' spectra, and (3) application of luminosity and emission-line velocity width criteria.

Because of the evolution of the project software during the early phases of the SDSS, spectra of quasars could be obtained on the basis of photometric measurements/target selection criteria that are not identical to the final products. The DR3 cata$\log$ was prepared using version 5.4 of the photometric pipeline code. (The differences between this release of the photometric software and previous versions are described in Abazajian et al. [2004].) These photometric measurements are referred to as BEST values; the measurements used during the target selection are denoted as TARGET values. Differences between TARGET and BEST arise when (1) the image data are processed with a 
new version of the software or (2) new image data replace the observations used for the target selection. See Paper II for a more extensive discussion of the differences between TARGET and BEST and the impact this has on the SDSS quasar catalogs.

The absolute magnitude limit ( $\S 3.3$ ) was imposed on the BEST photometry. However, we also report the TARGET photometry, which may be more useful when constructing statistical samples. This situation arises because small changes in the photometry, while leaving the density of quasars constant, can change the individual quasars that appear in the sample; thus, only the TARGET sample has sufficient spectroscopic completeness in terms of statistical analysis.

\subsection{Creation of the Quasar Candidate Database}

The construction of the DR3 Quasar Catalog began with four separate queries (three automated, one manual) to the SDSS Catalog Archive Server (CAS). ${ }^{30}$ These queries produced a set of objects that should contain all the quasars found by the SDSS; various automated and interactive culls were applied to this sample to create the final catalog.

Before describing the queries in detail, we must examine the definition of the term "primary object" within the SDSS object catalog, as it is relevant to some of the queries. The CAS defines a unique set of object detections in order to remove duplications (e.g., an object can be detected twice in the overlap area of neighboring runs). This unique set of objects is designated as "primary" in the database, and only "primary" objects are considered during target selection. (The remaining objects can be "secondary" or "family"; see $\S 4.7$ in Stoughton et al. [2002] for a definition of these terms.) Because of differences in the photometric pipeline between TARGET and BEST mentioned in the previous section, it is possible that the BEST object belonging to an existing spectrum is not designated "primary" or is missing from the BEST catalog altogether. This can occur when different data are used for TARGET and BEST or because of changes in the photometric pipeline, in particular the deblender (see $\S 4.4$ of Abazajian et al. 2004). In addition, a few plates cover sky area outside the nominal survey boundaries (the so-called bonus plates) ${ }^{31}$ all objects on these plates are nonprimary in the TARGET version, and objects that fall outside of the final survey boundaries remain nonprimary in BEST. The spectroscopic target selection flags and photometry for both BEST and TARGET processing are included in the catalog; the latter set is important for constructing statistical samples (see G. T. Richards et al. 2005 , in preparation).

Below we present a brief description of the four queries used to assemble the quasar candidate database. The actual text of the queries is given following each description. Since some minor changes to the DR3 database have been made since the start of the construction of the catalog (e.g., manually revised redshifts and spectral classifications), queries run on the latest DR3 database may return slightly different numbers of objects than quoted below.

1. The first query is the union of primary objects targeted as quasar candidates and primary objects not targeted as quasar candidates but whose spectra were classified by the SDSS spectroscopic software as QUASAR, had redshifts $\geq 0.6$, or were unidentified by the automated software (SDSS spectral class UNKNOWN). This query produced 130,119 objects; this is the vast majority (over $99.5 \%$ ) of the initial quasar database.

\footnotetext{
${ }^{30}$ See http://cas.sdss.org.

31 See http://www.sdss.org/dr3/products/spectra.
}

SELECT * FROM BESTDR3 . photoObjAll as p

left outer join SpecObjAll as $s$

on $\mathrm{p} . \mathrm{objID}=\mathrm{s}$. bestObjID

WHERE ( $(p \cdot$ mode $=1)$

AND ( (p.primTarget \& 0x0000001f) $>0$

OR ( (p.primTarget \& 0x0000001f) $=0$

$\operatorname{AND}(\mathrm{s} \cdot \mathrm{z}>=0.6 \mathrm{OR} \mathrm{s} \cdot \operatorname{specClass}$ in $(3,4,0))))$ )

2. The second query recovered 286 objects with QUASAR or UNKNOWN spectra that were mapped to a photometric object in TARGET but not in BEST (due to differences in deblending, etc., between the two pipelines).

SELECT * FROM BESTDR3. . SpecObjAll as s

join TARGDR3. .PhotoObjAll as $\mathrm{p}$

on p.objID = s.targetObjID

WHERE ( . bestObjID=0 AND (zWarning \& 0x00020000 =0)

AND (s.specClass in $(3,4,0))$ )

3. Our third query was designed to recover nonprimary objects with spectral classification of QUASAR or UNKNOWN. There are 251 such objects in the quasar database, mostly from the bonus plates. SELECT * FROM BESTDR3. . specObjAll as s

left outer join photoObjAll as $\mathrm{p}$

on $\mathrm{s}$. bestObjID $=\mathrm{p} . \mathrm{objID}$

WHERE ( ( $p$.mode in $(2,3)$ )

AND (s.specClass in $(3,4,0))$ )

4. The previous three automated queries missed 33 quasars from the DR1 catalog. Four of these objects were database glitches in DR1; the objects are definitely quasars, but it is not possible to properly map these spectra to the spectroscopic fibers, so we cannot be certain of these quasars' celestial coordinates. We were able to positively identify one of these four quasars, as it was discovered by the Large Bright Quasar Survey (Hewett et al. 1995), but the other three are lost. The remaining 29 objects were not targeted as quasars in BEST, and the software processing incorrectly did not classify the spectra as QUASAR or UNKNOWN; these objects were added into the quasar candidate database. The existence of these quasars suggests an $\simeq 0.2 \%$ incompleteness rate in our cataloging of quasars in post-DR1 data. Since this query simply retrieved information on specific DR1 catalog quasars not recovered by the above three queries, the text of this query is not given here.

In an ideal survey (e.g., one in which there was no repeat imaging, no area overlap, and no change in software), only the first query would be required.

Three automated cuts were made to the raw quasar database of approximately 131,000 candidates: (1) the over 58,000 objects targeted as quasars but whose spectra had not yet been obtained by the closing date of DR3 were deleted, (2) candidates classified with high confidence as "stars" and that had redshifts less than 0.002 were rejected ( 7647 objects), ${ }^{32}$ and (3) multiple observations of the same object (coordinate agreement better than $1^{\prime \prime}$ ) were resolved; the primary spectrum with the highest signal-tonoise ratio $(\mathrm{S} / \mathrm{N})$ was retained (this action deleted 1205 spectra). These culls produced a list of 63,614 unique quasar candidates.

\footnotetext{
32 After this paper was submitted, we realized we could estimate the number of star+quasar blends discarded in this step using FIRST counterparts. Two radiodetected star+quasar blends missing from the catalog were discovered (SDSS J092853.5+570735.3 at $z=1.67$ and SDSS J105115.8+464417.3 at $z=1.42$ ). Given that only $8 \%$ of catalog objects are detected by FIRST, we expect that $25_{-16}^{+33}$ additional FIRST-undetected quasars are missing from the catalog because of blending with stars. Overall, star+quasar blending appears to create a negligible incompleteness of $0.06_{-0.03}^{+0.07 \%}$.
} 


\subsection{Visual Examination of the Spectra}

The SDSS spectra of the remaining quasar candidates were manually inspected by several of the authors (D. P. S., P. B. H., G. T. R., M. A. S., D. V. B., and S. F. A.). This effort confirmed that the spectroscopic pipeline redshifts and classifications of the overwhelming majority of the objects are accurate. Several thousand objects were dropped from the list because they were obviously not quasars. (These objects tended to be low-S/N stars, unusual stars, and a mix of absorption line and narrow emission line galaxies.) Spectra for which redshifts could not be determined (low $\mathrm{S} / \mathrm{N}$ or subject to data processing difficulties) were also removed from the sample. This visual inspection resulted in the revisions of the redshifts of a few hundred quasars; this change in the redshift was usually quite substantial. Most of these corrections have been applied to the CAS.

About $1 \%$ of the entries in the catalog (a few hundred objects) are not "ironclad" classical quasars or lack absolutely certain redshifts. There are numerous "extreme BAL quasars" (see Hall et al. 2002, 2004); it is difficult if not impossible to apply the emission-line width criterion for these objects, but they are clearly of interest, have more in common with "typical" quasars than with narrow emission line galaxies, and have historically been included in quasar catalogs. We have included in the catalog all objects with BAL spectra that meet the $M_{i}<-22$ luminosity criterion. The spectra at the $\mathrm{S} / \mathrm{N}$ limit of the catalog occasionally yield likely but not certain redshifts. (Witness the revisions of the redshifts of a few objects in each edition of this series of papers; see $\S 5.1$.)

\subsection{Luminosity and Line Width Criteria}

As in Paper II, we adopt a luminosity limit of $M_{i}=-22.0$ for an $H_{0}=70 \mathrm{~km} \mathrm{~s}^{-1} \mathrm{Mpc}^{-1}, \Omega_{M}=0.3, \Omega_{\Lambda}=0.7$ cosmology (Spergel et al. 2003). The absolute magnitudes were calculated by correcting the $i$ measurement for Galactic extinction (using the maps of Schlegel et al. 1998) and assuming that the quasar spectral energy distribution in the ultraviolet-optical can be represented by a power law $\left(f_{\nu} \propto \nu^{\alpha}\right)$, where $\alpha=-0.5$ (Vanden Berk et al. 2001). This calculation ignores the contributions of emission lines and the observed distribution in continuum slopes. Emission lines can contribute several tenths of a magnitude to the $k$-correction (see Richards et al. 2001), and variations in the continuum slopes can introduce a magnitude or more of error into the calculation of the absolute magnitude, depending on the redshift. The absolute magnitudes will be particularly uncertain at redshifts near and above 5, at which the Ly $\alpha$ line (with a typical observed equivalent width of $\approx 500 \AA$ ) and strong Ly $\alpha$ forest absorption enter the $i$ bandpass.

Our catalog has a luminosity limit of $M_{i}=-22.0$, which is lower than the cutoff in most quasar catalogs (see Paper II for a discussion of this point). Objects near this limit can have an appreciable amount of contamination by starlight (the host galaxy). Although the SDSS photometric measurements in the catalog are based on the PSF magnitudes, the nucleus of the host galaxy can appreciably contribute to this measurement for the lowest luminosity entries in the catalog (see Hao et al. 2005). An object of $M_{i}=-22.0$ will reach the $i=19.1$ "low-redshift" selection limit at a redshift of $\approx 0.4$.

After visual inspection and application of the luminosity criterion had reduced the number of quasar candidates to under 50,000 objects, the remaining spectra were processed with an automated line-measuring routine. The spectra for objects whose maximum line width was less than $1000 \mathrm{~km} \mathrm{~s}^{-1}$ were visually examined; if the measurement was deemed to be an accurate reflection of the line (automated routines occasionally have spectacular failures when dealing with complex line profiles), the object was removed from the catalog. The resulting catalog contains 46,420 entries.

\section{CATALOG FORMAT}

The DR3 SDSS Quasar Catalog is available in three types offiles at the SDSS public Web site: (1) a standard ASCII file with fixedsize columns, (2) a g-zipped compressed version of the ASCII file (which is smaller than the uncompressed version by a factor of nearly 5), and (3) a binary FITS table format. The following description applies to the standard ASCII file. All files contain the same number of columns, but the storage of the numbers differs slightly in the ASCII and FITS formats; the FITS header contains all the required documentation. Table 1 provides a summary of the information contained in each of the columns in the catalog.

The standard ASCII catalog (Table 2) contains information on 46,420 quasars in a $19.8 \mathrm{MB}$ file. The DR3 format is similar to that of DR1; the major difference is the inclusion of some additional SDSS observational/processing material in the DR3 catalog.

The first 71 lines consist of catalog documentation; this is followed by 46,420 lines containing information on the quasars. There are 65 columns in each line; a summary of the information is given in Table 1. (The documentation in the ASCII catalog header is essentially an expansion of Table 1.) At least one space separates all the column entries, and, except for the first and last columns (SDSS and NED object names), all entries are reported in either floating point or integer format.

Notes on the catalog columns:

1. The DR3 object designation, given in the format SDSS Jhhmmss.ss+ddmmss.s; only the final 18 characters are listed in the catalog (i.e., the "SDSS J" for each entry is dropped).

$2-3$. The $\mathrm{J} 2000.0$ coordinates (right ascension and declination) in decimal degrees. The positions for the vast majority of the objects are accurate to $0.1 \mathrm{rms}$ or better in each coordinate; the largest expected errors are 0.'2 (see Pier et al. 2003). The SDSS coordinates are placed in the International Celestial Reference System, primarily through the USNO CCD Astrograph Catalog (Zacharias et al. 2000), and have an rms accuracy of 0 ".045 per coordinate.

4. The quasar redshifts. A total of 377 CAS redshifts were revised during our visual inspection. A detailed description of the redshift measurements is given in $\S 4.10$ of Stoughton et al. (2002). A comparison of 299 quasars observed at multiple epochs by the SDSS (Wilhite et al. 2005) finds an rms difference of 0.006 in the measured redshifts for a given object.

5-14. The DR3 PSF magnitudes and errors (not corrected for Galactic reddening) from BEST photometry (or, when BEST is unavailable, from TARGET photometry) for each object in the five SDSS filters. The effective wavelengths of the $u, g, r, i$, and $z$ bandpasses are 3541, 4653, 6147, 7461, and $8904 \AA$, respectively (for an $\alpha=-0.5$ power-law spectral energy distribution using the definition of effective wavelength given in Schneider et al. [1983]). The photometric measurements are reported in the natural system of the SDSS camera, and the magnitudes are normalized to the AB system (Oke \& Gunn 1983). The measurements are reported as asinh magnitudes (Lupton et al. 1999); see Paper II and Abazajian et al. (2004) for additional discussion and references for the accuracy of the photometric measurements. The TARGET photometric measurements are presented in columns (55)-(64).

15. The Galactic extinction in the $u$ band based on the maps of Schlegel et al. (1998). For an $R_{V}=3.1$ absorbing medium, the extinctions in the SDSS bands can be expressed as

$$
A_{x}=C_{x} E(B-V),
$$


TABLE 1

SDSS DR3 Quasar Catalog Format

\begin{tabular}{|c|c|c|}
\hline Column & Format & Description \\
\hline ....................... & A18 & SDSS DR3 designation hhmmss.ss+ddmmss.s (J2000.0) \\
\hline 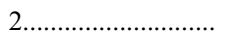 & F11.6 & Right ascension in decimal degrees (J2000.0) \\
\hline 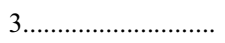 & F11.6 & Declination in decimal degrees $(\mathrm{J} 2000.0)$ \\
\hline 4 & F7.4 & Redshift \\
\hline 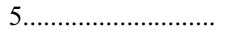 & F7.3 & PSF $u$ magnitude (not corrected for Galactic absorption) \\
\hline 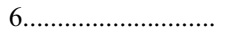 & F6.3 & Error in PSF $u$ magnitude \\
\hline 7 7............................... & F7.3 & PSF $g$ magnitude (not corrected for Galactic absorption) \\
\hline 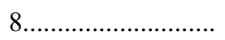 & F6.3 & Error in PSF $g$ magnitude \\
\hline 9 & F7.3 & PSF $r$ magnitude (not corrected for Galactic absorption) \\
\hline $10 \ldots \ldots \ldots \ldots \ldots \ldots \ldots \ldots . .$. & F6.3 & Error in PSF $r$ magnitude \\
\hline 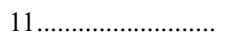 & F7.3 & PSF $i$ magnitude (not corrected for Galactic absorption) \\
\hline 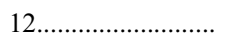 & F6.3 & Error in PSF $i$ magnitude \\
\hline 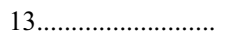 & F7.3 & PSF $z$ magnitude (not corrected for Galactic absorption) \\
\hline 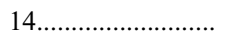 & F6.3 & Error in PSF $z$ magnitude \\
\hline 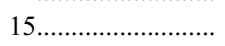 & F7.3 & Galactic absorption in $u$ band \\
\hline 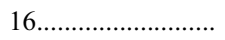 & F7.3 & $\log N_{\mathrm{H}}$ (logarithm of Galactic $\mathrm{H}$ I column density) \\
\hline 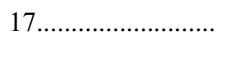 & F7.3 & $\begin{array}{l}\text { FIRST peak flux density at } 20 \mathrm{~cm} \text { expressed as AB magnitude; } \\
0.0 \text { is no detection, }-1.0 \text { indicates source is not in FIRST area }\end{array}$ \\
\hline 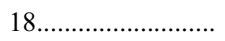 & F8.3 & $\mathrm{S} / \mathrm{N}$ of FIRST flux density \\
\hline 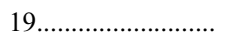 & F7.3 & SDSS-FIRST separation in arcseconds \\
\hline 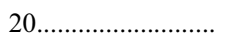 & I3 & $>3 \sigma$ FIRST flux at optical position but no FIRST counterpart within $2^{\prime \prime}$ ( 0 or 1 ) \\
\hline 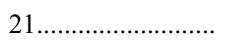 & $\mathrm{I} 3$ & FIRST source located $2^{\prime \prime}-30^{\prime \prime}$ from optical position $(0$ or 1$)$ \\
\hline 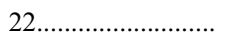 & F8.3 & log RASS full band count rate; -9.0 is no detection \\
\hline 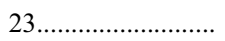 & F7.3 & $\mathrm{S} / \mathrm{N}$ of RASS count rate \\
\hline 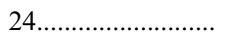 & F7.3 & SDSS-RASS separation in arcseconds \\
\hline 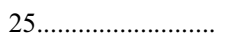 & F7.3 & $J$ magnitude (2MASS); 0.0 indicates no 2MASS detection \\
\hline 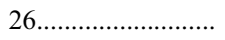 & F6.3 & Error in $J$ magnitude (2MASS) \\
\hline 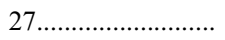 & F7.3 & $H$ magnitude (2MASS); 0.0 indicates no $2 \mathrm{MASS}$ detection \\
\hline 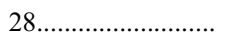 & F6.3 & Error in $H$ magnitude (2MASS) \\
\hline $29 \ldots \ldots \ldots \ldots \ldots \ldots \ldots \ldots \ldots$ & F7.3 & $K$ magnitude (2MASS); 0.0 indicates no 2 MASS detection \\
\hline 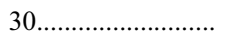 & F6.3 & Error in $K$ magnitude (2MASS) \\
\hline 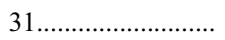 & F7.3 & SDSS-2MASS separation in arcseconds \\
\hline 32................................ & F8.3 & $M_{i}\left(H_{0}=70 \mathrm{~km} \mathrm{~s}^{-1} \mathrm{Mpc}^{-1}, \Omega_{M}=0.3, \Omega_{\Lambda}=0.7, \alpha_{\nu}=-0.5\right)$ \\
\hline 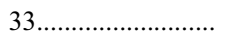 & $\mathrm{I} 3$ & Morphology flag; $0=$ point source, $1=$ extended \\
\hline 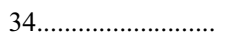 & $\mathrm{I} 3$ & SDSS SCIENCEPRIMARY flag (0 or 1$)$ \\
\hline $35 \ldots \ldots \ldots \ldots \ldots \ldots \ldots \ldots$ & $\mathrm{I} 3$ & SDSS MODE flag (blends, overlapping scans; 1,2 , or 3 ) \\
\hline 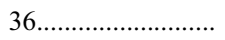 & $\mathrm{I} 12$ & Target selection flag (BEST) \\
\hline 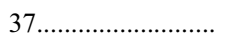 & $\mathrm{I} 3$ & Low- $z$ quasar selection flag ( 0 or 1$)$ \\
\hline 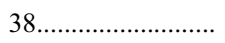 & $\mathrm{I} 3$ & High- $z$ quasar selection flag ( 0 or 1$)$ \\
\hline 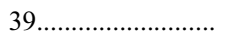 & $\mathrm{I} 3$ & FIRST selection flag ( 0 or 1$)$ \\
\hline 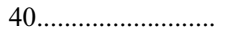 & $\mathrm{I} 3$ & ROSAT selection flag ( 0 or 1$)$ \\
\hline 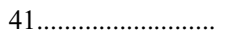 & $\mathrm{I} 3$ & Serendipity selection flag ( 0 or 1$)$ \\
\hline 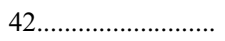 & $\mathrm{I} 3$ & Star selection flag ( 0 or 1$)$ \\
\hline 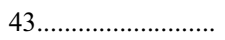 & $\mathrm{I} 3$ & Galaxy selection flag $(0$ or 1$)$ \\
\hline 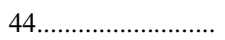 & I6 & SDSS imaging run number of photometric measurements \\
\hline 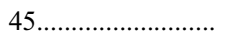 & I6 & MJD of imaging observation \\
\hline 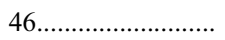 & I6 & MJD of spectroscopic observation \\
\hline 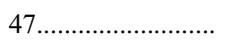 & $\mathrm{I} 5$ & Spectroscopic plate number \\
\hline 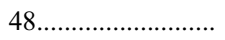 & $\mathrm{I} 5$ & Spectroscopic fiber number \\
\hline 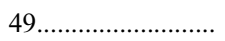 & $\mathrm{I} 4$ & SDSS photometric processing rerun number \\
\hline 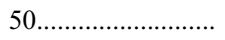 & $\mathrm{I} 3$ & SDSS camera column number \\
\hline 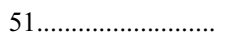 & $\mathrm{I} 5$ & SDSS frame number \\
\hline 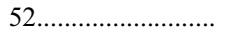 & I5 & SDSS object number \\
\hline $53 \ldots \ldots \ldots \ldots \ldots \ldots \ldots \ldots \ldots$ & I4 & SDSS chunk number \\
\hline 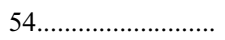 & I12 & Target selection flag (TARGET) \\
\hline 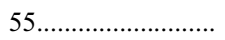 & F7.3 & TARGET PSF $u$ magnitude (not corrected for Galactic absorption) \\
\hline 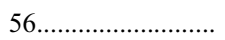 & F6.3 & TARGET error in PSF $u$ magnitude \\
\hline 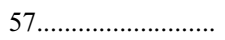 & F7.3 & TARGET PSF $g$ magnitude (not corrected for Galactic absorption) \\
\hline 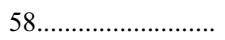 & F6.3 & TARGET error in PSF $g$ magnitude \\
\hline 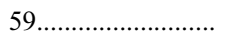 & F7.3 & TARGET PSF $r$ magnitude (not corrected for Galactic absorption) \\
\hline 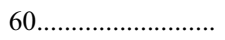 & F6.3 & TARGET error in PSF $r$ magnitude \\
\hline 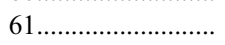 & F7.3 & TARGET PSF $i$ magnitude (not corrected for Galactic absorption) \\
\hline 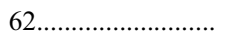 & F6.3 & TARGET error in PSF $i$ magnitude \\
\hline 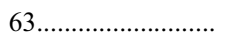 & F7.3 & TARGET PSF $z$ magnitude (not corrected for Galactic absorption) \\
\hline 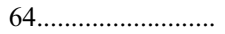 & F6.3 & TARGET error in PSF $z$ magnitude \\
\hline $65 \ldots \ldots \ldots \ldots \ldots \ldots \ldots \ldots \ldots$ & $1 \mathrm{X}, \mathrm{A} 25$ & Object name for previously known quasars; "SDSS" designates previously published SDSS object \\
\hline
\end{tabular}


TABLE 2

The SDSS Quasar Catalog III

\begin{tabular}{|c|c|c|c|c|c|c|c|c|c|}
\hline \multirow{2}{*}{$\frac{\text { Object (SDSS J) }}{000009.26+151754.5 \ldots \ldots \ldots \ldots \ldots . . .}$} & \multirow{2}{*}{$\begin{array}{c}\text { R.A. } \\
0.038605\end{array}$} & \multirow{2}{*}{$\begin{array}{c}\text { Decl. } \\
15.298476\end{array}$} & \multirow{2}{*}{$\frac{\text { Redshift }}{1.1986}$} & \multicolumn{2}{|c|}{$u$} & \multicolumn{2}{|c|}{$g$} & \multicolumn{2}{|c|}{$r$} \\
\hline & & & & 19.921 & 0.042 & 19.811 & 0.036 & 19.386 & 0.017 \\
\hline 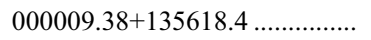 & 0.039088 & 13.938447 & 2.2400 & 19.218 & 0.026 & 18.893 & 0.022 & 18.445 & 0.018 \\
\hline $000009.42-102751.9 \ldots \ldots \ldots \ldots \ldots$ & 0.039269 & -10.464428 & 1.8442 & 19.249 & 0.036 & 19.029 & 0.027 & 18.980 & 0.021 \\
\hline $000011.41+145545.6 \ldots \ldots \ldots \ldots \ldots . .$. & 0.047547 & 14.929353 & 0.4596 & 19.637 & 0.030 & 19.466 & 0.024 & 19.362 & 0.022 \\
\hline 000011.96+000225.3 …............... & 0.049842 & 0.040372 & 0.4790 & 18.237 & 0.028 & 17.971 & 0.020 & 18.025 & 0.019 \\
\hline
\end{tabular}

Notes.-Table 2 is published in its entirety in the electronic edition of the Astronomical Journal. A portion is shown here for guidance regarding its form and content. The full catalog contains 65 columns of information on 46,420 quasars.

where $x$ is the filter (ugriz) and the values of $C_{x}$ are 5.155, $3.793,2.751,2.086$, and 1.479 for ugriz, respectively $\left(A_{g}, A_{r}\right.$, $A_{i}$, and $A_{z}$ are $0.736,0.534,0.405$, and 0.287 times $A_{u}$ ).

16. The logarithm of the Galactic neutral hydrogen column density along the line of sight to the quasar. These values were estimated via interpolation of the $21 \mathrm{~cm}$ data from Stark et al. (1992), using the COLDEN software provided by the Chandra $\mathrm{X}$-ray Center. Errors associated with the interpolation are typically expected to be less than $\approx 1 \times 10^{20} \mathrm{~cm}^{-2}$ (see $\S 5$ of Elvis et al. 1994).

17. Radio properties. If there is a source in the FIRST catalog within 2 ." 0 of the quasar position, this column contains the FIRST peak flux density at $20 \mathrm{~cm}$ encoded as an $\mathrm{AB}$ magnitude

$$
\mathrm{AB}=-2.5 \log \left(\frac{f_{\nu}}{3631 \mathrm{Jy}}\right)
$$

(see Ivezić et al. 2002). An entry of " 0.000 " indicates no match to a FIRST source; an entry of " -1.000 " indicates that the object does not lie in the region covered by the final catalog of the FIRST survey.

18. The $\mathrm{S} / \mathrm{N}$ of the FIRST source whose flux is given in column (17).

19. Separation between the SDSS and FIRST coordinates (in arcseconds).

20-21. Information about extended FIRST counterparts to the SDSS quasar so as to identify some of the potentially most interesting extended radio sources in the catalog.

In cases in which the FIRST counterpart to an SDSS source is extended, the FIRST catalog position of the source may differ by more than 2 " from the optical position. A " 1 " in column (20) indicates that no matching FIRST source was found within $2^{\prime \prime}$ of the optical position but that there is significant detection (larger than $3 \sigma$ ) of FIRST flux at the optical position. This is the case for 1319 SDSS quasars.

A " 1 " in column (21) identifies the 891 sources with a FIRST match in either column (17) or column (20) that also have at least one FIRST counterpart located between 2".0 (the SDSS-FIRST matching radius) and $30^{\prime \prime}$ of the optical position. Based on the average FIRST source surface density of $90 \mathrm{deg}^{-2}$, we expect $20-30$ of these matches to be chance superpositions.

22. The logarithm of the vignetting-corrected count rate (photons $\mathrm{s}^{-1}$ ) in the broad energy band $(0.1-2.4 \mathrm{keV}$ ) in the RASS Faint Source Catalog (Voges et al. 2000) and the RASS Bright Source Catalog (Voges et al. 1999). The matching radius was set to $30^{\prime \prime}$; an entry of " -9.000 " in this column indicates no $\mathrm{X}$-ray detection.

23. The $\mathrm{S} / \mathrm{N}$ of the ROSAT measurement.

24. Separation between the SDSS and RASS coordinates (in arcseconds).

25-30. The $J H K$ magnitudes and errors from the Two Micron All Sky Survey (2MASS) All-Sky Data Release Point Source
Catalog (Cutri et al. 2003) using a matching radius of 2".0. A nondetection by 2 MASS is indicated by a " 0.000 " in these columns. Note that the 2MASS measurements are Vega-based, not $\mathrm{AB}$, magnitudes.

31. Separation between the SDSS and 2MASS coordinates (in arcseconds).

32. The absolute magnitude in the $i$ band calculated by correcting for Galactic extinction and assuming $H_{0}=70 \mathrm{~km} \mathrm{~s}^{-1}$ $\mathrm{Mpc}^{-1}, \Omega_{M}=0.3, \Omega_{\Lambda}=0.7$, and a power-law (frequency) continuum index of -0.5 .

33. Morphological information. If the SDSS photometric pipeline classified the image of the quasar as a point source, the catalog entry is 0 ; if the quasar is extended, the catalog entry is 1 .

34. The SDSS SCIENCEPRIMARY flag, which indicates whether the spectrum was taken as a normal science spectrum (SCIENCEPRIMARY = 1) or for another purpose $($ SCIENCEPRIMARY $=0)$. The latter category contains quality assurance and calibration spectra, or spectra of objects located outside of the nominal survey area (e.g., bonus spectra; see $\S 3.1$ ).

35 . The SDSS MODE flag, which provides information on whether the object is designated primary (MODE $=1$ ), secondary $(\mathrm{MODE}=2$ ), or family (MODE $=3$ ). During target selection, only objects with MODE $=1$ are considered (except for objects on bonus plates); however, differences between TARGET and BEST photometric pipeline versions make it possible that the BEST photometric object belonging to a spectrum either is not detected at all or is a nonprimary object (see $\S 3.1$ ). Over $99.5 \%$ of the catalog entries are primary; 174 quasars are secondary, and six are family. For statistical analysis, users should restrict themselves to primary objects; secondary and family objects are included in the catalog for the sake of completeness with respect to confirmed quasars.

36 . The 32 bit SDSS target selection flag from BEST processing (PRIMTARGET; see Table 26 in Stoughton et al. [2002] for details). The target selection flag from TARGET processing is found in column (54).

37-43. The spectroscopic target selection status (BEST) for each object. The target selection flag in column (36) is decoded for seven groups: low-redshift quasar, high-redshift quasar, FIRST, ROSAT, serendipity, star, and galaxy; see Table 3 for a summary. An entry of " 1 " indicates that the object satisfied the given criterion (see Stoughton et al. 2002). Note that an object can be targeted by more than one selection algorithm.

44-45. The SDSS imaging run number and the Modified Julian Date (MJD) of the photometric observation used in the catalog. The MJD is given as an integer; all observations on a given night have the same integer MJD (and, because of the observatory's location, the same UT date). For example, imaging run 94 has an MJD of 51,075; this observation was taken on the night of 1998 September 19 (UT).

46-48. Information about the spectroscopic observation (MJD, spectroscopic plate number, and spectroscopic fiber 
TABLE 3

Spectroscopic Target Selection (BEST)

\begin{tabular}{|c|c|c|}
\hline Class & Selected & Sole Selection \\
\hline Low $z$...................... & 28831 & 8738 \\
\hline High $-z \ldots \ldots \ldots \ldots \ldots . . .$. & 9852 & 2286 \\
\hline FIRST ....................... & 2173 & 114 \\
\hline ROSAT ..................... & 3146 & 366 \\
\hline Serendipity ............... & 25565 & 9703 \\
\hline Star .......................... & 495 & 108 \\
\hline Galaxy ..................... & 417 & 57 \\
\hline
\end{tabular}

number) used to determine the redshift. These three numbers are unique for each spectrum and can be used to retrieve the digital spectra from the public SDSS database.

49-53. Additional SDSS processing information: the photometric processing rerun number, the camera column (1-6) containing the image of the object, the frame number of the run containing the object, the object identification number, and the "chunk" number (referred to as "tilerun" in the CAS) used to assign the target selection flag (see Stoughton et al. [2002] for descriptions of these parameters).

54. The 32 bit SDSS target selection flag from the TARGET processing.

55-64. The DR3 PSF magnitudes and errors (not corrected for Galactic reddening) from TARGET photometry. For 59 quasars, the $u$ TARGET information is missing from the CAS as a result of a software error; these objects have " 0.000 " entered for the $u$ TARGET values.

65. NED information. If there is a source in the NED quasar database within $5 . " 0$ of the quasar position, the NED object name is given in this column. The matching was done using the 45,526 objects in the NED quasar database as of 2004 August.

\section{CATALOG SUMMARY}

Of the 46,420 objects in the catalog, 44,221 were discovered by the SDSS, and 28,400 are presented here for the first time. (We classify an object as previously known if the NED Quasar Catalog contains a quasar within $5^{\prime \prime}$ of the SDSS position. Occasionally NED lists the SDSS designation for an object that was discovered earlier via another investigation; we have not attempted to correct these misattributions.) The catalog quasars span a wide range of properties: redshifts from 0.078 to 5.414 , $15.10<i<21.78$ (160 objects have $i>20.5$; only five have $i>21.0$ ), and $-30.2<M_{i}<-22.0$. The catalog contains 3761,2672 , and 6192 matches to the FIRST, RASS, and 2MASS catalogs, respectively. The RASS and 2MASS catalogs cover essentially all the DR1 area, but $4683(10 \%)$ of the entries in the DR3 catalog lie outside of the FIRST region.

Figure 2 displays the distribution of the DR3 quasars in the $i$-redshift plane. (The five objects with $i>21$ are not plotted.) Objects that NED indicates were previously discovered by investigations other than the SDSS are indicated with open circles. The curved cutoff on the left-hand side of the graph is due to the minimum luminosity criterion $\left(M_{i}<-22\right)$. The ridge in the contours at $i \approx 19.1$ for redshifts below 3 reflects the flux limit of the low-redshift sample; essentially all the large number of $z<3$ points with $i>19.1$ are quasars selected via criteria other than the primary multicolor sample.

A histogram of the catalog redshifts is shown in Figure 3. A clear majority of quasars have redshifts below 2 (the median redshift is 1.47 , the mode is $\approx 1.85)$, but there is a significant tail of objects out beyond a redshift of $5\left(z_{\max }=5.41\right)$. The dips in

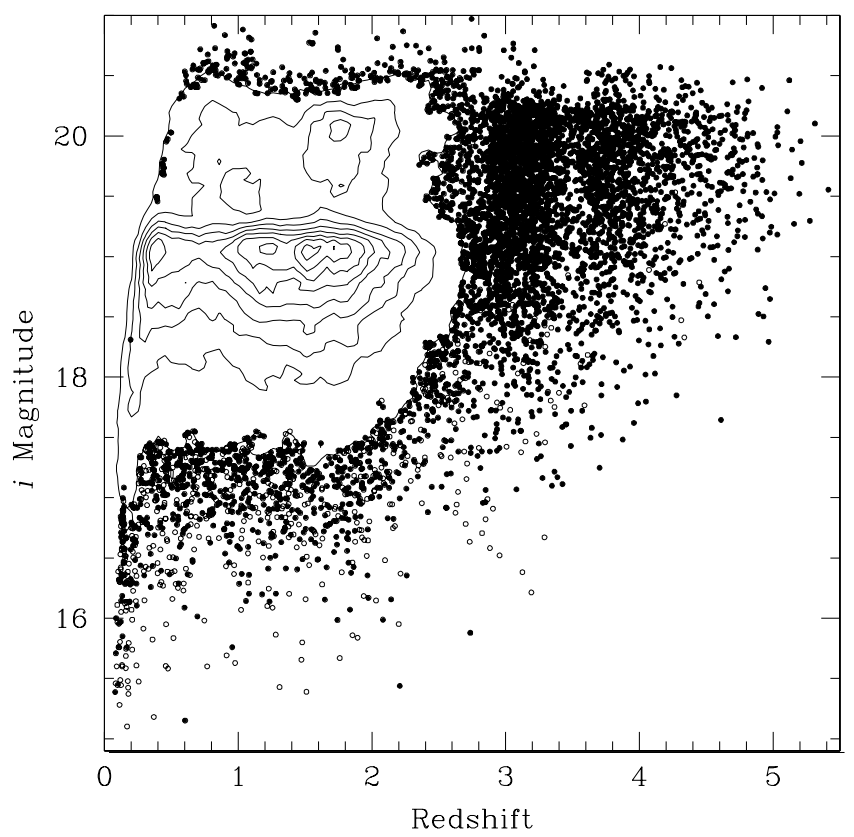

FIG. 2.-Observed $i$ magnitude as a function of redshift for the 46,420 objects in the catalog. Open circles indicate quasars in NED that were recovered but not discovered by the SDSS. Five quasars with $i>21$ are not plotted. The distribution is represented by a set of linear contours when the density of points in this two-dimensional space exceeds a certain threshold.

the curve at redshifts of 2.7 and 3.5 arise because the SDSS colors of quasars at these redshifts are similar to the colors of stars; we decided to accept significant incompleteness at these redshifts rather than be overwhelmed by a large number of stellar contaminants in the spectroscopic survey. Improvements in the quasar target selection algorithm since the previous edition of the SDSS Quasar Catalog have considerably increased the efficiency of target selection at redshifts near 3.5 (compare Fig. 3 with Paper II's Fig. 4; see Richards et al. [2002] for a discussion).

The distribution of the observed $i$ magnitude (not corrected for Galactic extinction) of the quasars is given in Figure 4. The sharp drops in the histogram at $i \approx 19.1$ and 20.2 are due to the magnitude limits in the low- and high-redshift samples, respectively.

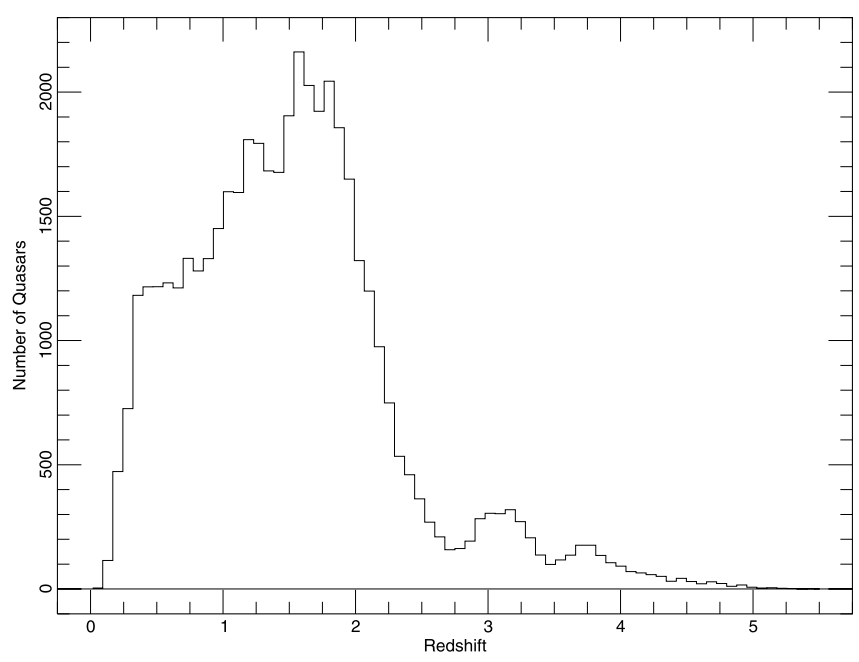

Fig. 3.-Redshift histogram of the catalog quasars. The smallest redshift is 0.08 , and the largest redshift is 5.41 ; the median redshift of the catalog is 1.47 . The redshift bins have a width of 0.076 . The dips at redshifts of 2.7 and 3.5 are caused by the lower efficiency of the selection algorithm at these redshifts. 


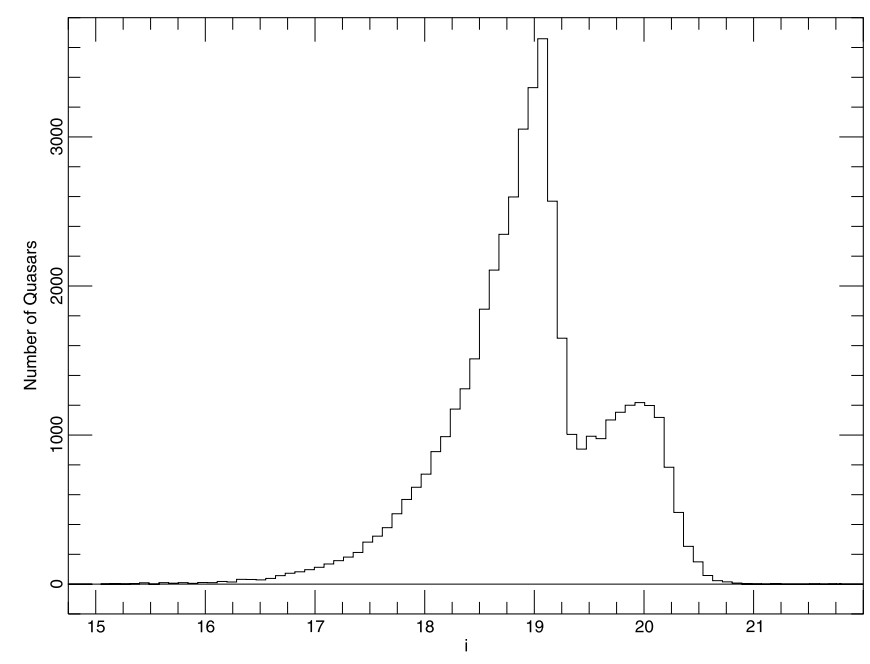

FIG. 4.-The $i$ magnitude (not corrected for Galactic absorption) histogram of the catalog quasars. The magnitude bins have a width of 0.089 . The sharp drop that occurs at magnitudes slightly fainter than 19 is due to the low-redshift flux limit of the survey. Quasars fainter than the $i=20.2$ high-redshift selection limit were found via other selection algorithms, primarily serendipity.

Figure 5 displays the distribution of the absolute $i$ magnitudes. There is a roughly symmetric peak centered at $M_{i}=-26$ with a FWHM of approximately 1 mag. The histogram drops off sharply at high luminosities (only $1.6 \%$ of the objects have $M_{i}<-28.0$ ) and has a gradual decline toward lower luminosities.

A summary of the spectroscopic selection is given in Table 3. We report seven selection classes in the catalog (cols. [37]-[43]). The second column in Table 3 gives the number of objects that satisfied a given selection criterion; the third column contains the number of objects that were identified only by that selection class. Slightly over two-thirds $(68 \%)$ of the catalog entries were selected on the basis of the SDSS quasar selection criteria (a lowredshift candidate, high-redshift candidate, or both). Approximately $55 \%$ of the quasars in the catalog are serendipity-flagged candidates, which is also primarily an "unusual color" algorithm; about one-fifth of the catalog was selected by the serendipity criterion alone.

Of the 31,403 DR3 quasars that have Galactic-absorptioncorrected $i$ magnitudes brighter than 19.1, 29,345 (93.4\%) were found from the quasar multicolor selection; if one combines the multicolor and FIRST selections (the primary quasar target selection criteria), all but 1777 of the $i<19.1$ objects are selected.

\subsection{Differences between the DR1 and DR3 SDSS Quasar Catalogs}

The DR1 catalog (Paper II) contains 16,713 objects. The DR3 coverage includes all the Paper II area, so one would expect that all the Paper II quasars would be included in the new edition. A comparison of the catalogs, defining a match as a positional coincidence of better than $1^{\prime \prime}$, reveals that 43 Paper II quasars $(0.26 \%)$ are missing in the new catalog. Each of these cases has been investigated; a summary of the results is given in Table 4. There are several reasons for the omissions:

1. Visual examination of the DR3-processed spectrum convinced us that either the object was not a quasar or the $\mathrm{S} / \mathrm{N}$ was insufficient to assign a redshift with confidence (15 DR1 quasars).

2. The widest line in the DR3-processed spectrum had a FWHM of less than $1000 \mathrm{~km} \mathrm{~s}^{-1}$ (14 DR1 quasars).

3. The luminosity of the object dropped below $M_{i}=-22$. This can arise because the latest processing produces new pho-

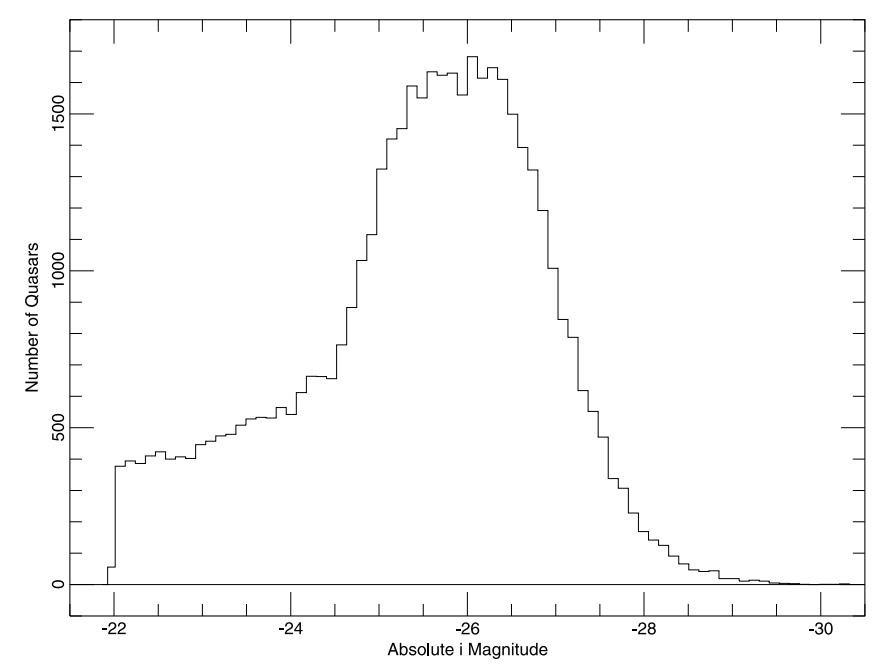

FIG. 5.-Luminosity distribution of the catalog quasars. The absolute magnitude bins have a width of 0.114 .

tometric measurements or because different imaging data are used between DR1 and DR3. (In addition to measurement errors, variability can play a role.) All the objects dropped for this reason were near the luminosity cutoff in the DR1 catalog (nine DR1 quasars).

4. Uncertain fiber mapping in the DR3 database forced us to drop three DR1 quasars. These objects are definitely quasars, but we are no longer certain (as we thought we were when using the DR1 database) of the celestial positions.

5. The positions of two DR1 quasars, whose spectra were taken on spectroscopic plate 540, changed by more than $1^{\prime \prime}$ (in these cases, 2.0 and 3.5) in the DR3 database.

Four of the entries in the DR3 catalog have redshifts that differ by more than 0.1 from the DR1 values. (The changes in redshift are large: $0.52,0.84,1.44$, and 2.33.) These quasars are reviewed in $\S 5.10$. Only seven quasars have $i$ measurements that differ by more than $0.5 \mathrm{mag}$ between DR 1 and DR3. In all cases the DR3 measurements are considered more reliable than those presented in previous publications.

\subsection{Bright Quasars}

Although the spectroscopic survey is limited to objects fainter than $i=15$, the SDSS continues to discover "PG class" (Schmidt $\&$ Green 1983) objects. The DR3 catalog contains 56 entries with $i<16.0$; seven were previously unknown. The spectra of the brightest two discoveries, SDSS J151921.66+590823.7 $(i=$ $15.39, z=0.078)$ and SDSS J152156.48+520238.5 $(i=15.44$, $z=2.21$ ), are presented in Figure 1. Six of the seven new bright quasars have redshifts below 0.2 and are in the low-luminosity tail of the catalog $\left(M_{i}>-24.0\right.$; see Fig. 5); however, the $z=$ 2.21 object is spectacularly luminous (see $\S 5.3$ ). A comparison of the SDSS and PG surveys is presented in Jester et al. (2005).

\subsection{Luminous Quasars}

There are 68 catalog quasars with $M_{i}<-29.0$ (3C 273 has $M_{i} \approx-26.6$ in our adopted cosmology); 25 are published here for the first time. HS 1700+6416 (=SDSS J170100.62+ 641209.0), at $M_{i}=-30.24$ and $z=2.74$, is the most luminous quasar in the catalog. Four objects have $M_{i}<-30.0$, including SDSS J152156.48+520238.5 (see previous section), which, at $M_{i}=-30.19$, is the third most luminous catalog entry. The spectrum of this object possesses a number of low equivalent width 
TABLE 4

DR1 Quasars Not in DR3 Quasar Catalog

\begin{tabular}{|c|c|c|c|}
\hline DR1 SDSS J & Comment & DR1 SDSS J & Comment \\
\hline $014349.15+002128.3 \ldots \ldots \ldots \ldots \ldots \ldots \ldots \ldots$ & Visual exam & $103951.49+643004.1 \ldots \ldots \ldots \ldots \ldots \ldots \ldots \ldots$ & $v<1000 \mathrm{~km} \mathrm{~s}^{-1}$ \\
\hline $021047.00-100153.0 \ldots \ldots \ldots \ldots \ldots \ldots \ldots \ldots$ & $v<1000 \mathrm{~km} \mathrm{~s}^{-1}$ & $104718.29+000718.4 \ldots \ldots \ldots \ldots \ldots \ldots \ldots \ldots \ldots \ldots \ldots \ldots \ldots \ldots$ & Visual exam \\
\hline $022011.27-000636.2 \ldots \ldots \ldots \ldots \ldots \ldots \ldots \ldots$ & $M_{i}>-22.0$ & $114245.45+680236.5 \ldots \ldots \ldots \ldots \ldots \ldots \ldots \ldots$ & $v<1000 \mathrm{~km} \mathrm{~s}^{-1}$ \\
\hline 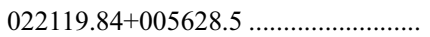 & $v<1000 \mathrm{~km} \mathrm{~s}^{-1}$ & $115404.55-001009.8 \ldots \ldots \ldots \ldots \ldots \ldots \ldots \ldots \ldots \ldots \ldots \ldots \ldots \ldots \ldots$ & Visual exam \\
\hline $024921.52-080957.1 \ldots \ldots \ldots \ldots \ldots \ldots \ldots \ldots$ & Unmapped fiber & $125751.28-004350.6 \ldots \ldots \ldots \ldots \ldots \ldots \ldots \ldots \ldots \ldots \ldots \ldots \ldots$ & $v<1000 \mathrm{~km} \mathrm{~s}^{-1}$ \\
\hline $025448.47-071735.3 \ldots \ldots \ldots \ldots \ldots \ldots \ldots$ & Visual exam & $132444.10-021746.5 \ldots \ldots \ldots \ldots \ldots \ldots \ldots$ & Visual exam \\
\hline $033606.70-000754.7 \ldots \ldots \ldots \ldots \ldots \ldots \ldots \ldots \ldots$ & Visual exam & 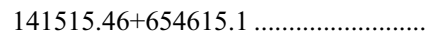 & $M_{i}>-22.0$ \\
\hline $080154.25+441233.9 \ldots \ldots \ldots \ldots \ldots \ldots \ldots \ldots$ & $v<1000 \mathrm{~km} \mathrm{~s}^{-1}$ & $142505.59+035336.2 \ldots \ldots \ldots \ldots \ldots \ldots \ldots \ldots \ldots \ldots \ldots \ldots \ldots \ldots \ldots$ & Visual exam \\
\hline $083223.22+491320.9 \ldots \ldots \ldots \ldots \ldots \ldots \ldots \ldots$ & Visual exam & 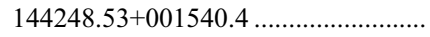 & Visual exam \\
\hline 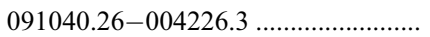 & Visual exam & $144454.60+004224.5 \ldots \ldots \ldots \ldots \ldots \ldots \ldots \ldots \ldots \ldots \ldots \ldots \ldots \ldots$ & $v<1000 \mathrm{~km} \mathrm{~s}^{-1}$ \\
\hline 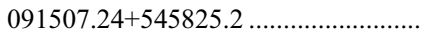 & $M_{i}>-22.0$ & 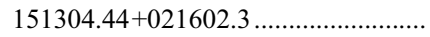 & $151304.35+021603.8$ \\
\hline 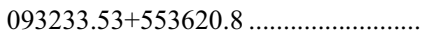 & Unmapped fiber & $151320.82+024128.5 \ldots \ldots \ldots \ldots \ldots \ldots \ldots \ldots \ldots \ldots \ldots \ldots \ldots \ldots$ & $151320.61+024130.1$ \\
\hline 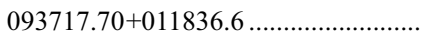 & $v<1000 \mathrm{~km} \mathrm{~s}^{-1}$ & $152200.77+035017.0 \ldots$ & $v<1000 \mathrm{~km} \mathrm{~s}^{-1}$ \\
\hline 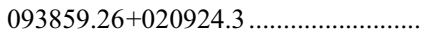 & Visual exam & 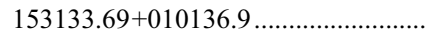 & Visual exam \\
\hline 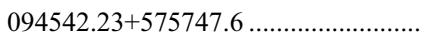 & Visual exam & $155936.46+025601.5 \ldots \ldots \ldots \ldots \ldots \ldots \ldots \ldots \ldots \ldots \ldots \ldots \ldots \ldots$ & $v<1000 \mathrm{~km} \mathrm{~s}^{-1}$ \\
\hline $094620.21+010452.0 \ldots \ldots \ldots \ldots \ldots \ldots \ldots \ldots$ & Visual exam & $163353.22+431111.0 \ldots \ldots \ldots \ldots \ldots \ldots \ldots \ldots \ldots \ldots \ldots \ldots \ldots \ldots$ & Visual exam \\
\hline 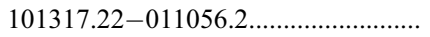 & $M_{i}>-22.0$ & 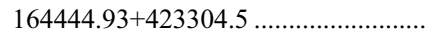 & $M_{i}>-22.0$ \\
\hline 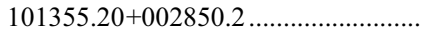 & $M_{i}>-22.0$ & 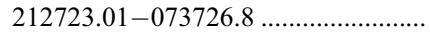 & Unmapped fiber \\
\hline $102423.28-004800.5 \ldots \ldots \ldots \ldots \ldots \ldots \ldots \ldots \ldots \ldots \ldots \ldots \ldots \ldots$ & $M_{i}>-22.0$ & $214828.94-064145.8 \ldots \ldots \ldots \ldots \ldots \ldots \ldots \ldots \ldots \ldots \ldots \ldots \ldots \ldots$ & $v<1000 \mathrm{~km} \mathrm{~s}^{-1}$ \\
\hline 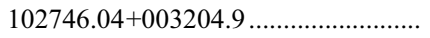 & $v<1000 \mathrm{~km} \mathrm{~s}^{-1}$ & $232137.82+011128.9 \ldots \ldots \ldots \ldots \ldots \ldots \ldots \ldots \ldots \ldots \ldots \ldots \ldots \ldots \ldots$ & $v<1000 \mathrm{~km} \mathrm{~s}^{-1}$ \\
\hline $102757.09+005406.9 \ldots \ldots \ldots \ldots \ldots \ldots \ldots \ldots \ldots \ldots \ldots \ldots \ldots$ & $M_{i}>-22.0$ & 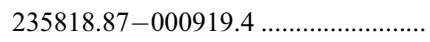 & $v<1000 \mathrm{~km} \mathrm{~s}^{-1}$ \\
\hline 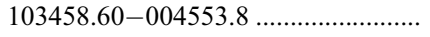 & $M_{i}>-22.0$ & & \\
\hline
\end{tabular}

emission lines, which is expected from the Baldwin (1977) effect. The image of the quasar is unresolved, so if it is lensed the image separation must be considerably less than $1^{\prime \prime}$ (see Pindor et al. 2003). This object is not seen in the FIRST or the RASS databases. The latter point might strike the reader as surprising given the brightness of the object. We can quantify the relationship between the optical brightness and the X-ray upper limit via the quantity $\alpha_{\text {ox }}$, the point-to-point spectral slope between rest frame $2500 \AA$ and $2 \mathrm{keV}$. For this object we find, adopting the assumptions in $\S 2$ of Brandt et al. (2002), that $\alpha_{\text {ox }} \leq-1.7$. This constraint is only moderately interesting; given the luminosity$\alpha_{\text {ox }}$ relation (e.g., Strateva et al. 2005), this quasar would be expected to have $\alpha_{\text {ox }}$ just below this limit. The lack of an X-ray detection could also be explained if the object were a BAL quasar (e.g., Brandt et al. 2000), but there is no evidence of any BAL features in the spectrum.

\subsection{Broad Absorption Line Quasars}

The SDSS quasar selection algorithm has proven to be effective at finding a wide variety of BAL quasars. A catalog of 224 BAL quasars drawn from the Paper I sample is given in Reichard et al. (2003); we are currently constructing a BAL quasar catalog, which will contain well over 1000 objects, from the 46,420 DR3 quasars (J. R. Trump et al. 2005, in preparation). BAL quasars are usually recognized by the presence of $\mathrm{C}$ IV absorption features, which are only visible in SDSS spectra at $z>1.6$; thus, the frequency of the BAL quasar phenomenon cannot be found from simply taking the ratio of BAL quasars to total number of quasars in the SDSS catalog. During the first few years of the SDSS a wide variety of "extreme BAL quasars" were discovered (see Hall et al. 2002); while the SDSS continues to find significant numbers of such objects (the spectrum of a new extreme BAL quasar is displayed in Fig. 1 [bottom right]), the DR3 catalog contains only two BAL quasar spectra that qualitatively differ from previously published types: a He II BAL quasar (SDSS J162805.81+474415.7) and a possible BAL quasar with a strange and unexplained continuum shape (SDSS J073816.91+
314437.1). Spectra of both of these BAL quasars are displayed in Hall et al. (2004).

\subsection{Quasars with Redshifts below 0.15}

The catalog contains 69 quasars with redshifts below $0.15 ; 30$ are presented here for the first time. All these objects are of low luminosity $\left(M_{i}>-23.5\right)$ because of the $i=15.0$ limit for the spectroscopic sample. Most of these quasars (53 out of 69) are extended in the SDSS image data. Figure 1 displays the spectra of the two lowest redshift quasars among the recent discoveries, SDSS J151921.66+590823.7 (also mentioned in $\S 5.2$ ) and SDSS J214054.55+002538.2; both have redshifts near 0.08 .

\subsection{High-Redshift ( $z \geq 4)$ Quasars}

One of the most exciting results produced by the SDSS is the identification of high-redshift quasars; the SDSS has discovered quasars out to a redshift of 6.4 (Fan et al. 2003 and references therein). Quasars with redshifts larger than $\approx 5.7$ cannot be found by the SDSS spectroscopic survey, because at these redshifts the observed wavelength of the Ly $\alpha$ emission line is redward of the $i$ band; at this point quasars become single-filter $(z)$ detections. At the typical $z$-band flux levels for redshift 6 quasars, there are simply too many "false positives" to undertake automated targeting. The largest redshift in the DR1 catalog is that of SDSS J023137.65-072854.5 at $z=5.41$, which was originally described by Anderson et al. (2001). (Indeed, since DR3 represents nearly half of the survey area, this result suggests that the effective redshift limit for the SDSS spectroscopic survey is nearer 5.5 than 5.7$)$.

The DR3 catalog contains 520 quasars with redshifts larger than 4; this is quite striking, since only a decade ago the published number of such objects was only about two dozen. The SDSS discovered 512 of these quasars; 322 are presented here for the first time. The catalog contains 17 quasars with redshifts above 5; spectra of the 12 new objects with the highest redshifts (all with redshifts greater than or equal to 4.99) are displayed in Figure 6 . 

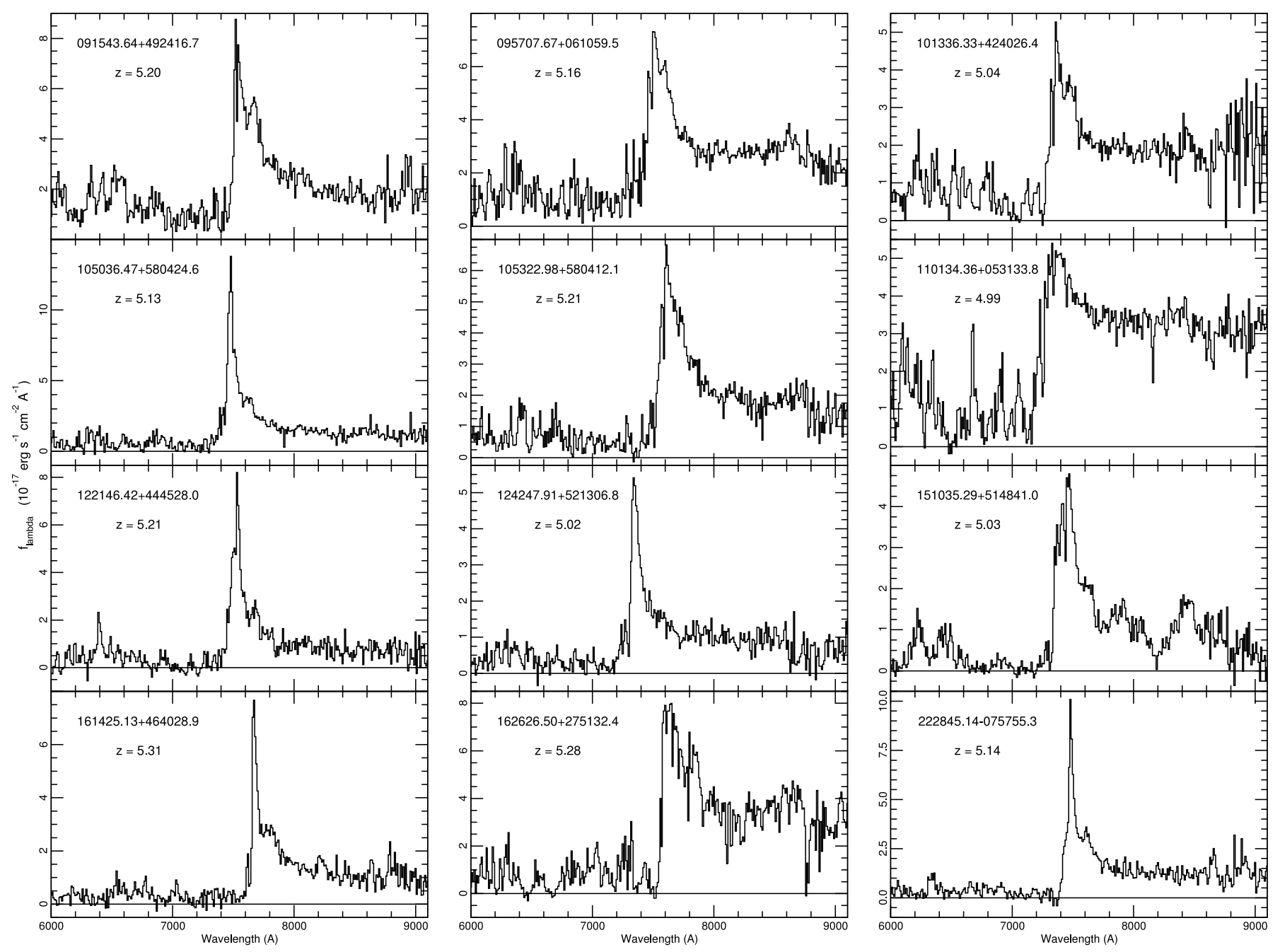

FIG. 6.-SDSS spectra of the 12 new quasars with the highest redshifts $(z \geq 4.99)$. The spectra have been rebinned to $10 \AA$ pixel ${ }^{-1}$ for display purposes. The wavelength region below $6000 \AA ̊$ has been removed because of the lack of signal in these objects.

The processed spectra for a few of the high-redshift quasars have gaps (usually caused by extreme contamination of the spectrum from bright, neighboring objects) that include of the region containing the Ly $\alpha$ emission line. The shapes of such spectra (in particular, the region associated with the Ly $\alpha$ forest), however, are so distinctive that we are confident that our redshift assignments are correct. To verify that this is an appropriate procedure, we obtained a spectrum with the Low Resolution Spectrograph (Hill et al. 1998) on the Hobby-Eberly Telescope (HET) of the highest redshift quasar with this defect in the SDSS spectrum: SDSS J162623.38+484136.4. The HET spectrum confirmed that this is indeed a redshift 4.9 quasar.

The flux limits of the RASS are such that only the most extreme X-ray sources can be detected at redshifts larger than 4 . We have checked for new X-ray detections in XMM-Newton, ROSAT (pointed observations), and Chandra data; no additional clear detections are found from the first two instruments, but six of the $z>4$ quasars in the catalog have previously unreported $\mathrm{X}$-ray detections in Chandra data. These detections have limited numbers of counts; thus, detailed X-ray spectral analyses are not possible. We have computed the quasars' point-to-point spectral slopes between rest frame $2500 \AA$ and $2 \mathrm{keV}\left(\alpha_{\text {ox }}\right)$, adopting the assumptions in $\S 2$ of Brandt et al. (2002). Considering the known dependence of $\alpha_{\text {ox }}$ on luminosity (e.g., Vignali et al. 2003a; Strateva et al. 2005), four of the quasars have X-ray emission at a nominal level for radio-quiet quasars: SDSS J102622.89+ $471907.0\left(\alpha_{\mathrm{ox}}=-1.59 ; z=4.94\right)$, SDSS J105322.98+580412.1 $\left(\alpha_{\mathrm{ox}}=-1.57 ; z=5.21\right)$, SDSS J222509.19-001406.8 $\left(\alpha_{\mathrm{ox}}=\right.$ $-1.79 ; z=4.89)$, and SDSS J222845.14-075755.3 $\left(\alpha_{\mathrm{ox}}=\right.$ $-1.78 ; z=5.14)$. These are some of the highest redshift X-ray detections obtained to date.

The remaining two quasars with Chandra detections have more remarkable X-ray properties. SDSS J001115.23+144601.8 $\left(\alpha_{\mathrm{ox}}=-1.28 ; z=4.96\right)$ is a radio-detected quasar $(37 \mathrm{mJy}$ at 1.4 GHz; Condon et al. 1998) that is notably X-ray bright. Its observed-frame $0.5-2 \mathrm{keV}$ flux is $1.0 \times 10^{-13} \mathrm{ergs} \mathrm{cm}^{-2} \mathrm{~s}^{-1}$, making it one of the X-ray-brightest objects known at $z>4$. The basic X-ray and radio properties of this quasar are similar to those of the handful of X-ray-luminous "blazars" studied at $z>4$ (see Table 3 of Bassett et al. [2004] and references therein). The relatively weak Ly $\alpha$ equivalent width of this quasar may be due to dilution by a beamed continuum. SDSS J144231.72+ $011055.2\left(\alpha_{\mathrm{ox}}=-1.37 ; z=4.51\right)$ is a weak-line quasar discussed in $\S 4$ of Anderson et al. (2001); the nature of weak-line quasars remains mysterious. Its relatively strong $\mathrm{X}$-ray emission suggests that a beamed X-ray continuum component may be present, although it is not a strong radio source. (Its integrated FIRST $20 \mathrm{~cm}$ flux density of $1.87 \mathrm{mJy}$ indicates that it is only moderately radio-loud.) The relatively strong $\mathrm{X}$-ray emission of SDSS J144231.72+011055.2 is notably different from that of 
SDSS J153259.96-003944.1 (Fan et al. 1999), the prototype SDSS weak-line quasar, which is fairly X-ray weak $\left(\alpha_{\text {ox }}<\right.$ -1.79 ; see Table A1 of Vignali et al. 2003b). There is apparently significant variety among the weak-line quasar population, even when one considers the time gap (up to several months in the rest frame) between the optical and X-ray observations.

\subsection{Close Pairs}

The mechanical constraint that SDSS spectroscopic fibers must be separated by $55^{\prime \prime}$ on a given plate makes it difficult for the spectroscopic survey to confirm close pairs of quasars. In regions that are covered by more than one plate, however, it is possible to obtain spectra of both components of a close pair; there are 121 pairs of quasars in the catalog with angular separation less than $60^{\prime \prime}$ (11 pairs with separations less than $20^{\prime \prime}$ ). Most of the pairs are chance superpositions, but there are seven pairs that may be physically associated systems $(\Delta z<0.02)$; they are listed in Table 5. Hennawi et al. (2005) identified over 200 physical quasar pairs, primarily through spectroscopic observations of unconfirmed SDSS quasar candidates near known SDSS quasars.

\subsection{Morphology}

The images of 2077 of the DR3 quasars are classified as extended by the SDSS photometric pipeline; 1961 (94\%) have
TABLE 5

Candidate Binary Quasars

\begin{tabular}{ccccc}
\hline \hline Quasar 1 & Quasar 2 & $z_{1}$ & $z_{2}$ & $\begin{array}{c}\Delta \theta \\
(\operatorname{arcsec})\end{array}$ \\
\hline $025959.68+004813.6$ & $030000.57+004828.0$ & 0.892 & 0.900 & 19.6 \\
$074336.85+205512.0$ & $074337.28+205437.1$ & 1.570 & 1.565 & 35.5 \\
$085625.63+511137.0$ & $085626.71+511117.8$ & 0.543 & 0.543 & 21.8 \\
$090923.12+000204.0$ & $090924.01+000211.0$ & 1.884 & 1.865 & 15.0 \\
$095556.37+061642.4$ & $095559.02+061701.8$ & 1.278 & 1.273 & 44.0 \\
$110357.71+031808.2$ & $110401.48+031817.5$ & 1.941 & 1.923 & 57.3 \\
$121840.47+501543.4$ & $121841.00+501535.8$ & 1.457 & 1.455 & 9.1 \\
\hline
\end{tabular}

redshifts below 1. (There are seven resolved $z>3.0$ quasars.) The majority of the large-redshift "resolved" quasars are probably measurement errors, but this sample probably also contains a mix of chance superpositions of quasars and foreground objects or possibly some small angle separation gravitational lenses. (Indeed, several lenses are present in the resolved quasar sample; see Paper II.)

\subsection{Matches with Nonoptical Catalogs}

The DR3 Quasar Catalog lists matches in the radio, X-ray, and infrared bands. We report radio measurements from the FIRST
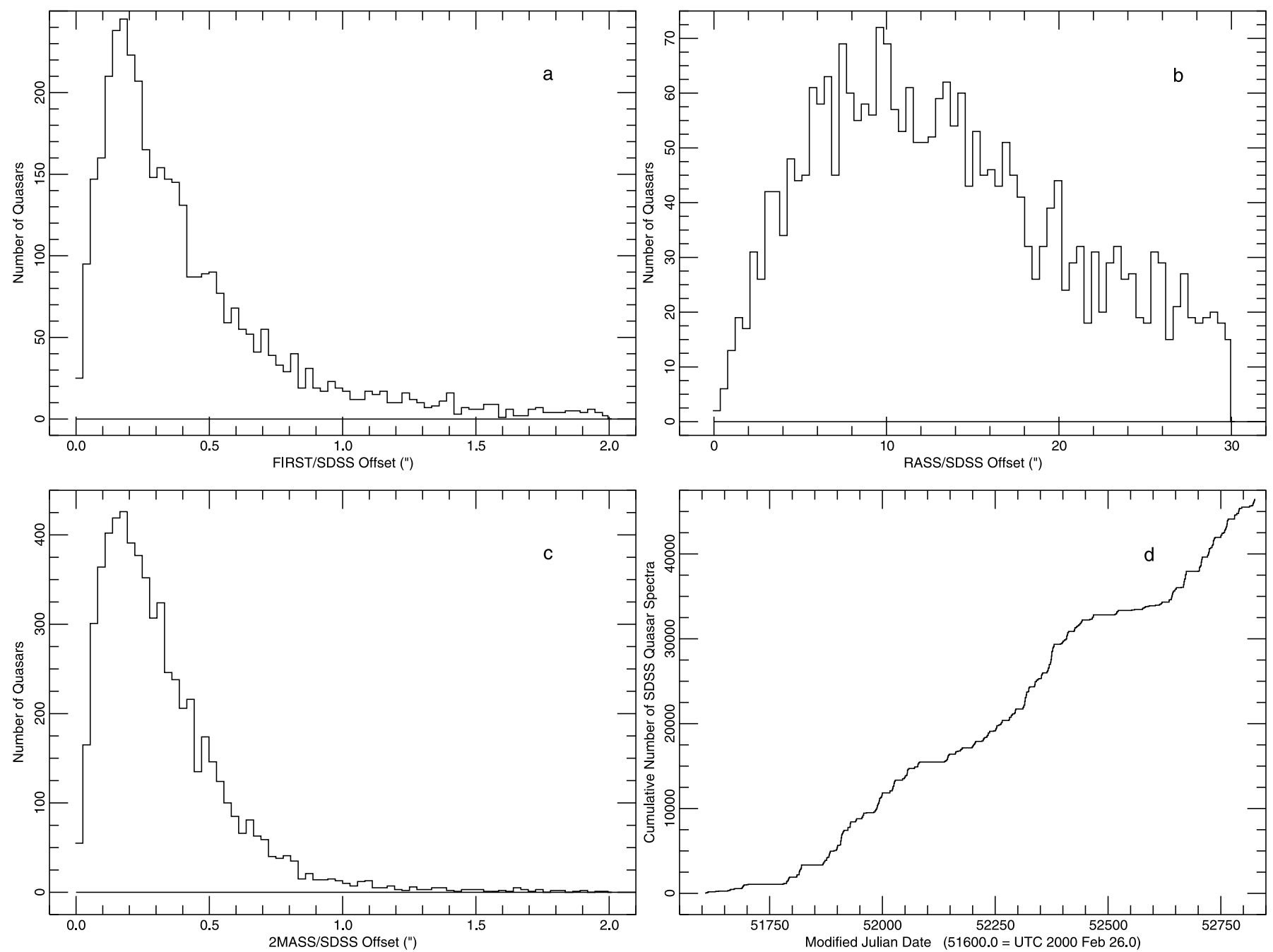

FIG. 7.- (a) Offsets between the 3761 SDSS and FIRST matches. The matching radius was set to 2".0. (b) Offsets between the 2672 SDSS and RASS Faint/Bright Source Catalog matches. The matching radius was set to $30^{\prime \prime}$. (c) Offsets between the 6192 SDSS and 2MASS matches. The matching radius was set to $2^{\prime \prime}$. (d) Cumulative number of DR3 quasars as a function of time. The horizontal axis runs from 2000 February to 2003 July. 
survey (Becker et al. 1995). A total of 3761 catalog objects are FIRST sources (defined by an SDSS-FIRST positional offset of less than 2."0). Extended radio sources may be missed by this matching; to recover at least some of these, we separately identify all objects with a greater than $3 \sigma$ detection of FIRST flux at the optical position (1319 sources). For these objects, as well as those with a FIRST catalog match within $2^{\prime \prime}$, we perform a second FIRST catalog search with $30^{\prime \prime}$ matching radius to identify possible radio lobes associated with the quasar, finding such matches for 891 sources.

Matches with the ROSAT All-Sky Survey Bright (Voges et al. 1999) and Faint (Voges et al. 2000) Source Catalogs were made with a maximum allowed positional offset of $30^{\prime \prime}$. The SDSS target selection for ROSAT sources initially considers SDSS ob-

TABLE 6

DR3-NED DiscREPANT REDSHIFTS

\begin{tabular}{|c|c|c|c|}
\hline SDSS J & $z_{\mathrm{DR} 3}$ & $z_{\mathrm{NED}}-z_{\mathrm{DR} 3}$ & NED Object Name ${ }^{a}$ \\
\hline $002411.65-004348.0 \ldots \ldots$. & 1.794 & -1.023 & LBQS $0021-0100$ \\
\hline $012428.09-001118.4 \ldots \ldots$. & 1.728 & -1.213 & SDSS \\
\hline $021249.59+003448.7 \ldots \ldots$. & 1.222 & -1.058 & SDSS \\
\hline $023044.81-004658.0 \ldots \ldots$ & 0.916 & +0.908 & SDSS \\
\hline $033305.32-053708.9 \ldots \ldots$ & 4.218 & -0.128 & SDSS \\
\hline $083148.29+463650.0 \ldots \ldots$. & 0.830 & +2.330 & SDSS \\
\hline $084035.41+412645.6 \ldots \ldots$. & 0.866 & +0.284 & 2MASSi J0840353+412645 \\
\hline $091446.26+400304.6 \ldots \ldots$. & 0.322 & +0.614 & NGC $2782 \mathrm{U} 1$ \\
\hline $092004.31+591732.7 \ldots \ldots$. & 1.286 & -0.724 & SBS 0916+595 \\
\hline $093052.25+003458.8 \ldots \ldots$. & 1.771 & -1.266 & [HB89] 0928+008 \\
\hline $094443.08+580953.2 \ldots \ldots$. & 0.561 & +0.146 & SBS $0941+583$ \\
\hline $095227.30+504850.6 \ldots \ldots$. & 1.091 & +0.455 & SBS $0949+510$ \\
\hline $095723.69+011458.7 \ldots \ldots$. & 2.466 & -1.543 & SDSS \\
\hline $101104.39+005724.0 \ldots \ldots$. & 0.683 & -0.417 & SDSS \\
\hline $101139.85+004039.5 \ldots \ldots$. & 1.712 & +0.606 & SDSS \\
\hline $101211.63+003719.4 \ldots \ldots$. & 1.631 & -0.891 & SDSS \\
\hline $103351.42+605107.3 \ldots \ldots$. & 1.401 & -1.065 & $87 \mathrm{~GB} 103034.2+610640$ \\
\hline $103506.01+565257.9 \ldots \ldots$. & 1.855 & -1.278 & $87 \mathrm{~GB} 103154.7+570825$ \\
\hline $103626.33+045436.4 \ldots \ldots$. & 1.049 & -0.667 & [CC91] 103350.4+051010 \\
\hline $104207.56+501321.9 \ldots \ldots$. & 1.265 & -0.915 & $4 C+50.31$ \\
\hline $104612.40+033624.9 \ldots \ldots$. & 2.112 & -0.632 & [CC91] 104337.3+035213 \\
\hline $111922.35+604851.3 \ldots \ldots \ldots$ & 2.014 & -1.716 & SBS $1116+610$ \\
\hline $113049.37-025048.2 \ldots \ldots$. & 1.408 & -0.670 & 2QZ J113049.3-025049 \\
\hline $114106.59-014107.5 \ldots \ldots$. & 1.422 & -0.152 & 2QZ J114106.5-014108 \\
\hline $114534.12+010308.0 \ldots \ldots$. & 1.076 & -0.674 & SDSS \\
\hline $120015.35+000553.1 \ldots \ldots$. & 1.650 & -1.281 & SDSS \\
\hline $124129.57+602041.2 \ldots \ldots$. & 2.069 & -0.612 & 2MASSi J1241296+602041 \\
\hline $125339.67+510203.7 \ldots \ldots$. & 1.565 & -0.810 & SBS $1251+513$ \\
\hline $130907.23+560326.6 \ldots \ldots$. & 2.155 & -2.138 & SBS $1307+563$ \\
\hline $133121.81+000248.4 \ldots \ldots$. & 3.218 & -2.352 & SDSS \\
\hline $134050.48-013449.2 \ldots \ldots$. & 0.744 & +0.726 & UM 600 \\
\hline $134809.71-013847.1 \ldots \ldots$ & 0.897 & +1.537 & 2QZ J134809.7-013848 \\
\hline $140846.46+552336.0 \ldots \ldots$. & 2.022 & -1.362 & SBS $1407+556$ \\
\hline $140848.81+650528.0 \ldots \ldots$. & 1.937 & -0.927 & SDSS \\
\hline $141454.73+552802.5 \ldots \ldots$. & 2.031 & -1.360 & SBS $1413+556$ \\
\hline $143233.96-012145.4 \ldots \ldots$ & 0.521 & +1.972 & 2QZ J143233.9-012145 \\
\hline $143601.58+002042.0 \ldots \ldots$. & 1.787 & -0.522 & SDSS \\
\hline $151307.26-000559.3 \ldots \ldots$ & 1.022 & +0.838 & SDSS \\
\hline $152529.92-001537.4 \ldots \ldots$. & 0.758 & +1.442 & SDSS \\
\hline $152715.82+491833.4 \ldots \ldots$. & 2.270 & -0.668 & SBS $1525+494$ \\
\hline $161351.34+374258.7 \ldots \ldots$. & 0.808 & +0.822 & [HB89] 1612+378 \\
\hline $162111.27+374604.9 \ldots \ldots \ldots$ & 1.273 & -0.933 & $4 \mathrm{C}+37.46$ \\
\hline $163647.07+385501.6 \ldots \ldots$. & 1.100 & +0.484 & [CCS88] 163503.7+390101 \\
\hline $163815.04+392731.9 \ldots \ldots$. & 0.573 & +0.717 & [CCS88] 163633.0+393322 \\
\hline $171930.24+584804.7 \ldots \ldots$. & 2.081 & -1.376 & SDSS \\
\hline
\end{tabular}

${ }^{\mathrm{a}}$ [CC91] = Clowes \& Campusano (1991); [CCS88] = Crampton et al. (1988); [HB89] = Hewett \& Burbidge (1989). jects that exceed the $30^{\prime \prime}$ catalog matching radius. The DR3 catalog lists a total of 2672 RASS matches.

The infrared information is provided by the 2MASS All-Sky Data Release Point Source Catalog (Cutri et al. 2003). The DR3 Quasar Catalog contains the $J H K$ photometric measurements of 6192 SDSS-2MASS matches (maximum positional offset of $2^{\prime \prime}$ ).

Figures $7 a-7 c$ show the distribution of the positional offsets for the FIRST, RASS, and 2MASS matches, respectively. The three histograms are quite similar in shape to the offset distributions found in Paper II. The number of chance superpositions between the DR3 Quasar Catalog and the FIRST and RASS data sets was estimated by shifting the quasar positions by $\pm 200^{\prime \prime}$. As was found in Paper II, virtually all the FIRST identifications are correct (an average of two FIRST "matches" was found after declination shifting), while approximately $1 \%$ of the ROSAT matches are misidentifications (an average of 20 ROSAT "matches" was found after shifting).

\subsection{Redshift Disagreements with Previous Measurements}

The redshifts of 45 quasars in this catalog disagree by more than 0.10 from the values given in the NED database; the information for each of these objects is given in Table 6. (Four of the entries are differences between the DR1 and DR3 quasar catalogs.) A NED name of "SDSS" indicates that the NED entry is taken from a previous SDSS publication. The relatively large number of apparent discrepancies with previous SDSS measurements arises because the NED redshift for these objects is frequently the redshift given in the SDSS data release and not from the quasar catalogs. For example, the second entry in Table 6 was included in both the EDR and DR1 quasar catalogs with the correct redshift, but the NED value was the redshift reported in the EDR itself. In every case we believe that the redshifts quoted in this catalog are more consistent with the SDSS spectra than are the NED values.

\section{FUTURE WORK}

The 46,420 quasars were identified from $\approx 40 \%$ of the proposed SDSS survey area. The progress of the SDSS quasar survey can be seen in Figure $7 d$, which displays the cumulative number of SDSS quasars as a function of observing date. There are yearly "plateaus" in this figure that coincide with late summer/ fall; at this time of the year the north Galactic pole region is unavailable. The primary spectroscopic survey of the south Galactic pole is now complete; observations in this region now consist of additional imaging scans (to reach fainter magnitudes; see York et al. 2000) and a series of specialized spectroscopic programs (e.g., empirical determination of the effectiveness of the SDSS quasar selection; Vanden Berk et al. 2005).

Investigations of the quasar luminosity function and the spatial distribution of quasars based on SDSS data are given in G. T. Richards et al. (2005, in preparation) and Yahata et al. (2005). We plan to publish the next edition of the SDSS quasar catalog with the SDSS Fifth Data Release, which is currently expected to occur in 2006.

We thank the referee, Buell Jannuzi, for a number of suggestions that improved the paper. This work was supported in part by National Science Foundation grants AST 03-07582 (D. P. S., D. V. B.), AST 03-07384 (X. F.), and AST 03-07409 (M. A. S.) and by NASA LTSA grant NAG5-13035 and CXC grant GO34117X (W. N. B., D. P. S.). X. F. acknowledges support from an Alfred P. Sloan Fellowship and a David and Lucile Packard Fellowship in Science and Engineering. S. J. and C. S. were 
supported by the US Department of Energy under contract DEAC02-76CH03000.

Funding for the creation and distribution of the SDSS Archive has been provided by the Alfred P. Sloan Foundation, the Participating Institutions, the National Aeronautics and Space Administration, the National Science Foundation, the US Department of Energy, the Japanese Monbukagakusho, and the Max Planck Society. The SDSS Web site is http://www.sdss.org. The SDSS is managed by the Astrophysical Research Consortium for the Participating Institutions. The Participating Institutions are the University of Chicago, Fermilab, the Institute for Advanced Study, the Japan Participation Group, The Johns Hopkins University, the Korean Scientist Group, Los Alamos National Laboratory, the Max Planck Institute for Astronomy, the Max Planck Institute for Astrophysics, New Mexico State University, the University of Pittsburgh, the University of Portsmouth, Princeton University, the United States Naval Observatory, and the University of Washington.
This research has made use of (1) the NED, which is operated by the Jet Propulsion Laboratory, California Institute of Technology, under contract with the National Aeronautics and Space Administration, and (2) data products from 2MASS, which is a joint project of the University of Massachusetts and the Infrared Processing and Analysis Center, California Institute of Technology, funded by the National Aeronautics and Space Administration and the National Science Foundation.

The HET is a joint project of the University of Texas at Austin, Pennsylvania State University, Stanford University, Ludwig-Maximillians-Universität München, and Georg-AugustUniversität Göttingen. The HET is named in honor of its principal benefactors, William P. Hobby and Robert E. Eberly. The Marcario Low Resolution Spectrograph is named for Mike Marcario of High Lonesome Optics, who fabricated several optics for the instrument but died before its completion; it is a joint project of the HET partnership and the Instituto de Astronomía de la Universidad Nacional Autónoma de México.
Abazajian, K., et al. 2003, AJ, 126, 2081

2004, AJ, 128, 502 2005, AJ, 129, 1755

Anderson, S. F., et al. 2001, AJ, 122, 503 2003, AJ, 126, 2209

Baldwin, J. A. 1977, ApJ, 214, 679

Bassett, L. C., Brandt, W. N., Schneider, D. P., Vignali, C., Chartas, G., \& Garmire, G. P. 2004, AJ, 128, 523

Becker, R. H., White, R. L., \& Helfand, D. J. 1995, ApJ, 450, 559

Blanton, M. R., Lupton, R. H., Maley, F. M., Young, N., Zehavi, I., \& Loveday, J. 2003, AJ, 125, 2276

Brandt, W. N., Laor, A., \& Wills, B. J. 2000, ApJ, 528, 637

Brandt, W. N., et al. 2002, ApJ, 569, L5

Castander, F. J., et al. 2001, AJ, 121, 2331

Clowes, R. G., \& Campusano, L. E. 1991, MNRAS, 249, 218

Collinge, M., et al. 2005, AJ, 129, 2542

Condon, J. J., Cotton, W. D., Greisen, E. W., Yin, Q. F., Perley, R. A., Taylor, G. B., \& Broderick, J. J. 1998, AJ, 115, 1693

Crampton, D., Cowley, A. P., Schmidtke, P. C., Janson, T., \& Durrell, P. 1988, AJ, 96, 816

Croom, S. M., Smith, R. J., Boyle, B. J., Shanks, T., Miller, L., Outram, P. J., \& Loaring, N. S. 2004, MNRAS, 349, 1397

Cutri, R. M., et al. 2003, 2MASS All-Sky Catalog of Point Sources (Pasadena: IPAC)

Eisenstein, D. J., et al. 2001, AJ, 122, 2267

Elvis, M., Lockman, F. J., \& Fassnacht, C. 1994, ApJS, 95, 413

Fan, X., et al. 1999, ApJ, 526, L57 2003, AJ, 125, 1649

Fukugita, M., Ichikawa, T., Gunn, J. E., Doi, M., Shimasaku, K., \& Schneider, D. P. 1996, AJ, 111, 1748

Gunn, J. E., et al. 1998, AJ, 116, 3040

Hall, P. B., et al. 2002, ApJS, 141, 267 2004, in Multiwavelength AGN Surveys, ed. R. Mujica \& R. Maiolino (Singapore: World Scientific), 247

Hao, L., et al. 2005, AJ, 129, 1783

Hennawi, J., et al. 2005, AJ, submitted

Hewett, A., \& Burbidge, G. 1989, ApJS, 69, 1

Hewett, P. C., Foltz, C. B., \& Chaffee, F. H. 1995, AJ, 109, 1498

Hill, G. J., Nicklas, H. E., MacQueen, P. J., Mitsch, W., Wellem, W., Altmann, W., Wesley, G. L., \& Ray, F. B. 1998, Proc. SPIE, 3355, 433

Hogg, D. W., Schlegel, D. J., Finkbeiner, D. P., \& Gunn, J. E. 2001, AJ, 122, 2129
Ivezić, Ž., et al. 2002, AJ, 124, 2364 2004, Astron. Nachr., 325, 583

Jester, S., et al. 2005, AJ, submitted

Kauffmann, G., et al. 2003, MNRAS, 346, 1055

Lupton, R. H., Gunn, J. E., Ivezić, Ž., Knapp, G. R., Kent, S., \& Yasuda, N. 2001, in ASP Conf. Ser. 238, Astronomical Data Analysis Software and Systems X, ed. F. R. Harnden, F. A. Primini, \& H. E. Payne (San Francisco: ASP), 269

Lupton, R. H., Gunn, J. E., \& Szalay, A. 1999, AJ, 118, 1406

Oke, J. B., \& Gunn, J. E. 1983, ApJ, 266, 713

Pier, J. R., Munn, J. A., Hindsley, R. B., Hennessy, G. S., Kent, S. M., Lupton, R. H., \& Ivezić, Ž. 2003, AJ, 125, 1559

Pindor, B., Turner, E. L., Lupton, R. H., \& Brinkmann, J. 2003, AJ, 125, 2325

Reichard, T. A., et al. 2003, AJ, 125, 1711

Richards, G. T., et al. 2001, AJ, 121, 2308 2002, AJ, 123, 2945

Schlegel, D. J., Finkbeiner, D. P., \& Davis, M. 1998, ApJ, 500, 525

Schmidt, M., \& Green, R. F. 1983, ApJ, 269, 352

Schneider, D. P., Gunn, J. E., \& Hoessel, J. G. 1983, ApJ, 264, 337

Schneider, D. P., et al. 2002, AJ, 123, 567 (Paper I) 2003, AJ, 126, 2579 (Paper II)

Smith, J. A., et al. 2002, AJ, 123, 2121

Spergel, D. N., et al. 2003, ApJS, 148, 175

Stark, A. A., Gammie, C. F., Wilson, R. W., Bally, J., Linke, R. A., Heiles, C., \& Hurwitz, M. 1992, ApJS, 79, 77

Stoughton, C., et al. 2002, AJ, 123, 485

Strateva, I. V., Brandt, W. N., Schneider, D. P., Vanden Berk, D. E., \& Vignali, C. 2005, AJ, in press

Strauss, M. A., et al. 2002, AJ, 124, 1810

Vanden Berk, D. E., et al. 2001, AJ, 122, 549 2005, AJ, 129, 2047

Vignali, C., Brandt, W. N., \& Schneider, D. P. 2003a, AJ, 125, 433

Vignali, C., et al. 2003b, AJ, 125, 2876

Voges, W., et al. 1999, A\&A, 349, 389 2000, IAU Circ., 7432, 1

Wilhite, B. C., et al. 2005, AJ, in press

Yahata, K., et al. 2005, PASJ, submitted

York, D. G., et al. 2000, AJ, 120, 1579

Zacharias, N., et al. 2000, AJ, 120, 2131

Zakamska, N. L., et al. 2003, AJ, 126, 2125 